\title{
Existence and uniqueness of Lipschitz continuous graphs with prescribed Levi curvature ${ }^{\text {si }}$
}

\section{Existence et unicité des graphes Lipschitz continus à courbure de Levi prescrite}

\author{
Francesca Da Lio ${ }^{\mathrm{a}, *}$, Annamaria Montanari ${ }^{\mathrm{b}, 1}$ \\ ${ }^{a}$ Dipartimento di Matematica, Università di Torino, Via Carlo Alberto 10, 10123 Torino, Italy \\ b Dipartimento di Matematica, Università di Bologna, piazza di Porta S. Donato 5, 40127 Bologna, Italy
}

Received 19 January 2004; accepted 27 October 2004

Available online 7 April 2005

\begin{abstract}
In this paper we prove comparison principles between viscosity semicontinuous sub- and supersolutions of the generalized Dirichlet problem (in the sense of viscosity solutions) for the Levi Monge-Ampère equation. As a consequence of this result and of the Perron's method we get the existence of a continuous solution of the Dirichlet problem related to the prescribed Levi curvature equation under suitable assumptions on the boundary data and on the Levi curvature of the domain. We also show that such a solution is Lipschitz continuous by building Lipschitz continuous barriers and by applying a weak Bernstein method introduced by Barles in [Differential Integral Equations 4 (2) (1991) 241].

(C) 2006 L'Association Publications de l'Institut Henri Poincaré. Published by Elsevier B.V. All rights reserved
\end{abstract}

\section{Résumé}

Dans cet article, nous prouvons des principes de comparaison entre sous et sursolutions du problème de Dirichlet généralisé (dans le sens des solutions de viscosité) pour l'équation de Levi Monge-Ampère . Comme conséquence de ces résultats, nous obtenons l'existence d'une solution continue du problème de Dirichlet associé à l'équation de la courbure de Levi sous des hypothèses convenables sur les conditions au bord et sur l'ouvert. Nous prouvons que la solution est lipschitzienne par la méthode de Bernstein faible introduite par Barles dans [Differential Integral Equations 4 (2) (1991) 241].

() 2006 L'Association Publications de l'Institut Henri Poincaré. Published by Elsevier B.V. All rights reserved

MSC: 35J70; 35B50; 49L25

\footnotetext{
This work is partially supported by M.I.U.R., project "Viscosity, metric, and control theoretic methods for nonlinear partial differential equations".

* Corresponding author.

E-mail addresses: francesca.dalio@unito.it (F. Da Lio), montanar@dm.unibo.it (A. Montanari).

1 The second author was partially supported by University of Bologna. Funds for selected research topics.
}

0294-1449/\$ - see front matter (C) 2006 L'Association Publications de l'Institut Henri Poincaré. Published by Elsevier B.V. All rights reserved doi:10.1016/j.anihpc.2004.10.006 


\section{Introduction}

If $M$ is a hypersurface in $\mathbb{R}^{n}$, and if $\Pi$ is its second fundamental form, then the eigenvalues of $\Pi$ are the principal curvatures of $M$. The trace of $\Pi$ is called the mean curvature of $M$ and the determinant of $\Pi$ is the Gauss-Kronecker curvature. The Dirichlet problem for a convex graph with prescribed curvature is classical (see for example [13]). It has been considered by many authors in the past (see [24] for a list of references), starting from the pioneering works by A.D. Aleksandrov and I.Ya. Bakelman. For a real hypersurface $M \subset \mathbb{C}^{n+1}$, let $H$ denote the $n$-dimensional complex subspace of the tangent space to $M$. The restriction of the second fundamental form of $M$ on $H$ is a Hermitian form $\Lambda$, which is called the Levi form. More precisely, if $M$ is a real manifold of class $C^{2}$ which is locally defined by $\rho$, then the Levi form $\Lambda(\rho)$ is the restriction to the complex tangent space $H$ of the Hermitian form associated with the complex Hessian matrix $H \operatorname{ess}_{\mathbb{C}} \rho=\left(\frac{\partial^{2} \rho}{\partial z_{\ell} \partial \bar{z}_{p}}\right)_{\ell, p=1}^{n+1}$ of $\rho$. The Levi form is of great importance in the study of envelopes of holomorphy in the theory of holomorphic functions in $\mathbb{C}^{n+1}$ (see $[11,14,18,21]$ for details on this matter). By using the biholomorphic invariant analogue of Euclidean convexity (see for example [18]), it can be shown that the Levi form is the biholomorphic invariant part of the real Hessian of the defining function. Since $\Lambda$ is obtained from part of the second fundamental form of $M$, one can expect that it will have some properties similar to curvatures. However, $\Lambda$ itself depends on the defining function for the domain. This obstacle can be avoided as follows. If $M$ is given locally as $\{\rho=0\}$ with $\partial \rho \neq 0$, then one can define the normalized Levi form as $L(\rho)=\frac{\Lambda(\rho)}{|\partial \rho|}$. Easy calculations show that $L$ is independent of the defining function $\rho$ and depends only on the domain (a proof of this assertion can be found in [19, Proposition A.1]). Bedford and Gaveau [6] were the first to remark this fact and they used the normalized Levi form to bound the domain over which $M$ can be defined as the graph of a function of class $C^{2}$. The signature of $L$ is a biholomorphic invariant of $M$, although $L$ itself is not invariant. We recall that a domain $\{\rho<0\}$ is pseudoconvex (strongly pseudoconvex) if the Levi form $\rho$ (or equivalently the normalized Levi form) is semidefinite positive (positive definite) on the boundary. The eigenvalues of $L$ correspond to mean curvatures in certain complex directions and, more generally, symmetric functions in the eigenvalues of $L$ are complex curvatures of $M$. The product of the eigenvalues of $L$, corresponding to the complex version of the Gauss-Kronecker curvature of $M$, is the scalar function $k_{M}(\cdot)$ defined by

$$
k_{M}(z)=-|\partial \rho(z)|^{-n-2} \operatorname{det}\left(\begin{array}{cccc}
0 & \partial_{\overline{1}} \rho(z) & \cdots & \partial_{\overline{n+1}} \rho(z) \\
\partial_{1} \rho(z) & \partial_{1 \overline{1}} \rho(z) & \cdots & \partial_{1 \overline{n+1}} \rho(z) \\
\vdots & \vdots & \ddots & \vdots \\
\partial_{n+1} \rho(z) & \partial_{n+1 \overline{1}} \rho(z) & \cdots & \partial_{n+1 \overline{n+1}} \rho(z)
\end{array}\right) .
$$

We will call $k_{M}(z)$ the total Levi curvature of $M$ at a point $z \in M$. In (1.1) $\partial_{j}, \partial_{\bar{j}}, \partial_{\ell \bar{j}}$ denote respectively the derivatives $\frac{\partial}{\partial z_{j}}, \frac{\partial}{\partial \bar{z}_{j}}, \frac{\partial^{2}}{\partial z_{\ell} \partial \bar{z}_{j}}$ and $\partial \rho=\left(\partial_{1} \rho, \ldots, \partial_{n+1} \rho\right)$. To convince the reader that the total Levi curvature is the analogous of the Gauss curvature for the complex structure, we propose the following example.

Example 1.1 (Total Levi curvature of a ball). If $M$ is the ball of radius $r$ with center at zero, then by choosing as defining function $\rho=\left|z_{1}\right|^{2}+\cdots+\left|z_{n+1}\right|^{2}-r^{2}$, an easy calculation shows $k_{M} \equiv r^{-n}$.

However, a cylinder in $\mathbb{C}^{n+1}$ may not have zero total Levi curvature, as the following example shows.

Example 1.2 (Total Levi curvature of a cylinder). Let $B(0, r) \subset \mathbb{C}^{n} \times \mathbb{R}$ be a ball of radius $r$. We consider the following cylinder

$$
B(0, r) \times \mathrm{i} \mathbb{R}=\left\{(z, w) \in \mathbb{C}^{n} \times \mathbb{C}:|z|^{2}+\left(\frac{w+\bar{w}}{2}\right)^{2}-r^{2}<0\right\} .
$$


It is easy to check that

$$
\frac{1}{2 r^{n}} \leqslant k_{\partial B(0, r) \times i \mathbb{R}}(z, w)=\frac{r^{2}+(\operatorname{Re} w)^{2}}{2 r^{n+2}} \leqslant \frac{1}{r^{n}}
$$

for every $(z, w) \in \partial B(0, r) \times \mathrm{i} \mathbb{R}$.

If $M$ is the graph of a function $u: \Omega \rightarrow \mathbb{R}, \Omega \subset \mathbb{C}^{n} \times \mathbb{R}$, we say that $u$ is Levi convex in $\Omega$ if epi $(u)=\{(z, w) \in$ $\left.\mathbb{C}^{n+1}: \operatorname{Im} w>u(z, \operatorname{Re} w)\right\}$ is pseudoconvex at every point of $M$. In this situation for every $(z, w) \in \operatorname{epi}(u)$ we have

$$
k_{M}(z, w)=-2^{n+2}\left(1+|D u|^{2}\right)^{-(n+2) / 2} \operatorname{det}\left(\begin{array}{ccc}
0 & u_{\bar{z}} & u_{\operatorname{Re} w}-\mathrm{i} / 2 \\
u_{z} & u_{z \bar{z}} & u_{z \bar{w}} \\
u_{\operatorname{Re} w}+\mathrm{i} / 2 & u_{w \bar{z}} & u_{w \bar{w}}
\end{array}\right)
$$

where all the partial derivatives of $u$ are computed at $(z, \operatorname{Re} w)$. The determinant on the right-hand side is often called the Levi Monge-Ampère operator LMA(u), to emphasize the comparison with the Euclidean MongeAmpère operator. Even if the Levi curvature has some geometric properties similar to the Euclidean Gauss curvature we must stress that the Levi Monge-Ampère operator is never strictly elliptic, not even on the class of strictly convex functions. In this paper we consider the Dirichlet problem of finding a non parametric hypersurface with prescribed total Levi curvature $k$ on a domain $\Omega \subset \mathbb{C}^{n} \times \mathbb{R} \subset \mathbb{C}^{n} \times \mathbb{C}$ where $\Omega \times \mathrm{i} \mathbb{R}$ is strongly pseudoconvex. The problem can be formulated as follows. Given $\varphi \in C(\partial \Omega)$ and $k \geqslant 0$ continuous, find $u \in C(\bar{\Omega})$ Levi convex such that

$$
\left.u\right|_{\partial \Omega}=\varphi \quad \text { and } \quad \operatorname{LMA}(u)=k(\cdot, u)\left(1+|D u|^{2}\right)^{(n+2) / 2} \quad \text { on } \Omega .
$$

In the sequel we denote by $D u$ and $D^{2} u$ the gradient and the Hessian matrix of $u$ respectively. The Dirichlet problem for LMA for $n=1$ was considered first by A. Debiard and Gaveau [12], who gave an estimate for the modulus of continuity of the solution and by Z. Slodkowski and G. Tomassini in [22].

Z. Slodkowski and G. Tomassini defined in [23] viscosity solutions of the Dirichlet problem (1.2) with (1+ $|D u|^{2}$ ) raised to the $3 n / 2$ power. The technique developed in [23] is to reduce (1.2) to a Bellman problem for a family of quasilinear degenerate elliptic operator $L_{v}$ and to provide a priori estimates of the solutions of the uniformly elliptic equation

$$
L_{v}(u)+\varepsilon \Delta u=k^{1 / n}\left(1+|D u|^{2}\right)^{1 / 2}
$$

independent of $\varepsilon$ and of $v$. The main result is the existence of a Lipschitz continuous viscosity solution $u$ to (1.2). However, the method in [23] requires very strong conditions on $k$ and on the growth of its first and second derivatives (see [23, Theorem 2.4 and condition (2.5), p. 488]). In addition such a solution is shown to be unique only in the particular case $k \equiv 0$. We reacll that if $u$ is the solution of (1.2) with $k \equiv 0$, then for $\lambda \geqslant \max _{\Omega} u$, the set $\Gamma_{+}^{\lambda}(u)=\{(x, \mathrm{i} s) \in \bar{\Omega} \times \mathrm{i} \mathbb{R}: u(x) \leqslant s \leqslant \lambda\}$ is both the holomorphic hull and the envelope of holomorphy of $C_{\varphi, \lambda}=(\bar{\Omega} \times\{\mathrm{i} \lambda\}) \cup\{(x, \mathrm{i} s) \in \partial \Omega \times \mathrm{i} \mathbb{R}: \varphi(x) \leqslant s \leqslant \lambda\}$. In this paper we consider also the case $k \not \equiv 0$ which is seems to be significative above all from the point of view of the regularity theory of pde's. In [20] it is shown that LMA is degenerate elliptic in the set of Levi convex functions, namely, if $u, v$ are Levi convex and $D^{2} u \leqslant D^{2} v$ then $0 \leqslant L(u) \leqslant L(v)$ and $\operatorname{LMA}(u) \leqslant \operatorname{LMA}(v)$. Therefore one cannot expect in general $C^{\infty}$ regularity result for this equation. We notice that if $k \equiv 0$ then every real function of the last variable $u(\operatorname{Re} w)$ is a solution $\operatorname{LMA}(u)=0$. Hence, in this case the regularity of a solution comes from the regularity of the boundary data. However, if $k \neq 0$ the missing ellipticity direction can be recovered by taking into account the CR structure of the hypersurface. This fact has been used by F. Lascialfari and the second author in [20] to prove that the Levi Monge Ampère operator 
has some hypoelliptic properties analogous to the Monge-Ampère operator. Precisely, if $C^{m, \alpha}$ denote the usual Hölder space with respect to the Euclidean metric, the following regularity result was proved in [20].

Theorem. Let $\Omega \subset \mathbb{R}^{2 n+1}$ be an open set and $q \in C^{\infty}\left(\Omega \times \mathbb{R} \times \mathbb{R}^{2 n+1}\right), q>0$. If $u \in C^{2, \alpha}(\Omega)$ is a strictly Levi convex solution to the Levi Monge-Ampère equation

$$
\operatorname{LMA}(u)=q(\cdot, u, D u)
$$

then $u \in C^{\infty}(\Omega)$.

The existence of classical solution of Eq. (1.3) for $n>1$ is an interesting open problem while for $n=1$ it has been solved by Citti, Lanconelli and the second author in [7]. The main aim of this paper is to show the existence and the uniqueness of a Lipschitz continuous viscosity solution of (1.2) under far less restrictive regularity assumptions on the prescribed function $k$. To this purpose we use the main tools of the theory of viscosity solutions. We recall that the theory of viscosity solutions, which was initiated in the early 80 's by the paper of M.G. Crandall and P.L. Lions [8] not only provides a convenient partial differential equations framework for dealing with the lack of the existence of classical solutions, but also leads to the correct formulation of the "generalized" boundary conditions of fully nonlinear elliptic and parabolic pde's. For a complete survey of the results obtained within the theory of viscosity solutions for the first-order case we refer to the books of Bardi and Capuzzo Dolcetta [1] and Barles [2], while for the second-order case we refer to the "User's guide" of Crandall, Ishii and Lions [9].

In framework of viscosity solutions the standard Dirichlet boundary conditions have to be relaxed and read in the viscosity sense as

$$
\min \left(-\operatorname{LMA}(u)+2^{n} k(\cdot, u)\left(1+|D u|^{2}\right)^{(n+2) / 2}, u-\varphi\right) \leqslant 0 \quad \text { on } \partial \Omega
$$

and

$$
\max \left(-\operatorname{LMA}(u)+2^{n} k(\cdot, u)\left(1+|D u|^{2}\right)^{(n+2) / 2}, u-\varphi\right) \geqslant 0 \quad \text { on } \partial \Omega .
$$

Roughly speaking, these relaxed conditions mean that the equations has to hold up to the boundary, when the boundary condition is not assumed in the classical sense.

One of the main tools to prove the existence and the uniqueness of a continuous solution to (1.2) is to provide a comparison principle between semicontinuous sub and supersolutions to (1.2). Indeed the existence follows easily through the Perron's method by Ishii [15] with the version up to the boundary obtained by the first author in [10].

Hereafter we suppose that $\Omega \subset \mathbb{R}^{2 n+1}$ is a bounded domain with boundary of class $C^{2}$. We list below some basic assumptions we use throughout the paper.

We assume that $k: \bar{\Omega} \times \mathbb{R} \rightarrow[0,+\infty)$ is a continuous bounded function satisfying

(H1) for all $R>0$, there exists $\ell_{R}>0$, such that, for every $x \in \bar{\Omega}$, and $-R \leqslant v \leqslant u \leqslant R$

$$
\ell_{R}(u-v) \leqslant k^{1 / n}(\cdot, u)-k^{1 / n}(\cdot, v),
$$

(H2) for all $R>0$, for all $(x, y) \in \bar{\Omega}$ and $|u| \leqslant R$, there exists a modulus of continuity $\omega_{R}$ such that $\omega_{R}(s) \rightarrow 0$ as $s \rightarrow 0^{+}$and

$$
\left|k^{1 / n}(x, u)-k^{1 / n}(y, u)\right| \leqslant \omega_{R}(|x-y|)
$$

Conditions (H1) and (H2) will be used in Section 3 to prove a comparison principle between viscosity semicontinuous sub- and supersolution to the problem (1.2). In Section 4 to solve the Dirichlet problem by using the Perron's method we will use the following additional assumptions on $k$ and $\Omega$ :

(H3) $\Omega \times \mathrm{i} \mathbb{R}$ is strongly pseudoconvex and, for all $\xi_{0} \in \partial \Omega$, $\sup _{\bar{\Omega} \times \mathbb{R}} k<k_{\partial \Omega \times \mathrm{i}}\left(\xi_{0}\right)$.

(H4) $\sup _{\bar{\Omega} \times \mathbb{R}} k<1 / r^{n}$, where $r$ is the radius of the minimum sphere containing $\bar{\Omega}$. 
Condition (H3) will guarantee that there is no loss of boundary condition and will also allow to build local barriers to the problem (1.2). Condition (H4) will be used to build a particular global subsolution to (1.2) and thus it will permit to get the existence of a continuous solution by the Perron's method.

We prove the following theorems.

Theorem 1.1 (The strictly monotone case). Assume (H1)-(H4) hold. Then for any $\varphi \in C(\partial \Omega)$ there exists a unique continuous viscosity solution $u$ of (1.2). Moreover, if $k \in C^{0,1}(\bar{\Omega} \times W)$ for every $W \subset \subset \mathbb{R}$, and $\varphi \in C^{1,1}(\partial \Omega)$, then $u \in C^{0,1}(\bar{\Omega})$.

In order to include the case when the prescribed function $k$ is constant, (H1) may be modified and relaxed to

(H5) for all $R>0$, for every $x \in \bar{\Omega}$, and $-R \leqslant v \leqslant u \leqslant R$

$$
0 \leqslant k(\cdot, u)-k(\cdot, v) .
$$

Indeed, when $k$ is constant in $x$, i.e.

(H6) $k(x, u)=k(u)$ for all $(x, u) \in \Omega \times \mathbb{R}$,

the result can be strengthened as follows.

Theorem 1.2 (The $x$-independent case). Assume that (H2)-(H6) hold. Then, for every $\varphi \in C(\partial \Omega)$, there exists a unique continuous viscosity solution $u$ of (1.2). Moreover, if $\varphi \in C^{1,1}(\partial \Omega)$, then $u \in C^{0,1}(\bar{\Omega})$.

The proofs of Theorems 1.1 and 1.2 follow classical arguments from the theory of viscosity solutions (see e.g. [9]). The Lipschitz continuity of the solution is obtained by building local barriers on the boundary and by adapting to our setting the weak Bernstein method, which was introduced by Barles in [3] to get gradient bound for viscosity solutions to fully nonlinear degenerate elliptic pde's.

If in addition the prescribed function $k$ satisfies

(H7) $k \in C^{0,1}(\bar{\Omega} \times W)$ for every $W \subset \subset \mathbb{R}$,

and

(H8) there are $\alpha \geqslant 0, L>0$ such that

$$
\frac{D_{x} k \cdot p+D_{u} k|p|^{2}}{\left(1+|p|^{2}\right)^{1 / 2}}+g n k^{1+1 / n} \geqslant \alpha
$$

for almost every $(x, u) \in \Omega \times \mathbb{R}$ and for all $|p| \geqslant L$, for some $g \leqslant g_{0}$, where $g_{0}$ is the universal constant $\sqrt{2}(2-\sqrt{2})(\sqrt{2}+1)^{-1}$,

we prove the existence of a solution of (1.2) by an approximation argument. More precisely, by using local barriers and the weak Bernstein method, we get a priori estimates of the Lipschitz constant and of the $L_{\infty}$-norm of the solution of the approximating problem. The result is

Theorem 1.3 (The Lipschitz continuous case). Assume (H3)-(H5) and (H7)-(H8) hold. For every $\varphi \in C^{1,1}(\partial \Omega)$ there exists a Lipschitz continuous viscosity solution $u$ of (1.2). Moreover, if $k>0$, then the solution is unique. 
We should stress that for general fully nonlinear pde's the weak Bernstein method requires the inequality in (1.8) to hold for some $\alpha>0$. Because of the particular structure of the Levi Monge-Ampère operator here the constant $\alpha$ can be zero.

The uniqueness statement in Theorem 1.3 is obtained via a comparison principle between continuous sub- and supersolutions. In the case $k>0$ a strong comparison principle between $C^{2}$ sub- and supersolutions has been proved in [19], by using the fact that the nonellipticity direction can be recovered by commutations.

Our paper is organized as follows. In Section 2 we give a precise viscosity formulation of the Dirichlet problem (1.2) and we show the equivalence with the one given in [23]. In Section 3 we analyze the loss of boundary conditions for the Dirichlet problem (1.2). The question of loss of boundary conditions have been addressed by the first author in [10] for general fully nonlinear second order degenerate elliptic and parabolic equations. As it is well known, this fact may depend on various aspects such as the geometry of the domains, the structural properties of the operator appearing in the equation and the value of the boundary data. The main result of this section is that under the hypothesis (H3) there is no loss of boundary condition for the Dirichlet problem (1.2). In Section 4 we prove comparison principles between viscosity semicontinuous sub- and supersolutions to the problem (1.2) assuming either conditions (H1) and (H2), or (H2) and (H5)-(H6). Using a geometric property of the Levi curvature in this section we also prove a comparison principle between continuous sub- and supersolution for Lipschitz continuous $k>0$ satisfying (H7). This result yields the uniqueness of a solution of problem (1.2) in Theorem 1.3. As a byproduct of the comparison results and the Perron's method, under the hypothesis (H4) we get the existence of a continuous solution to (1.2) for all continuous boundary data. In Section 5, we show the existence of a Lipschitz continuous solution to (1.2). Moreover by using an approximation argument, together with some a priori estimate for the Lipschitz constant of a solution, we also prove the existence part of Theorem 1.3. In Section 6 we give an estimate of the maximum ball contained in $\Omega$ for which the problem (1.2) is solvable in the class of Lipschitz continuous viscosity solutions. Our argument is inspired from [6, Theorem 1] and to [19, Corollary 1.1]. Moreover, when the domain $\Omega$ is a ball, we shall prove a nonexistence result which shows that conditions (H3) and (H4) cannot be significantly relaxed.

\section{Graphs with prescribed Levi curvature in a viscosity sense}

In this section we give the definition of pseudoconvex domains and Levi convex functions in a suitable weak sense. We also give a precise formulation of the Dirichlet problem (1.2) in a viscosity sense.

We start with the following

Definition 2.1. An open set $D \subset \mathbb{C}^{n+1}$ is pseudoconvex in a generalized sense if for every $z_{0} \in \partial D$ and for every $\phi \in C^{2}\left(\mathbb{C}^{n+1}\right)$ such that $\partial_{z} \phi\left(z_{0}\right) \neq 0$ and $\left\{\phi(z)<\phi\left(z_{0}\right)\right\} \subseteq D$ near $z_{0}$, we have $L(\phi)\left(z_{0}\right) \geqslant 0$.

One can see that Definition 2.1 is equivalent to the definition of Hartogs pseudoconvexity given in the literature (see e.g. [18]). More precisely we have the following equivalences.

Proposition 2.1. Let $D \subset \mathbb{C}^{n+1}$ be an open set. The following conditions are equivalent:

(1) D is pseudoconvex in a generalized viscosity sense;

(2) For every $z_{0} \in \partial D$ and for every quadratic polynomial $q$ with $q\left(z_{0}\right)=0, \partial_{z} q\left(z_{0}\right) \neq 0$, such that $\{z: q(z)<0\}$ is contained in $D$ near $z_{0}$, then $L(q)\left(z_{0}\right) \geqslant 0$;

(3) D is Hartogs pseudoconvex.

Proof. The proof of Proposition 2.1 is implicitly contained in [17, Theorem 4.1.27]) and we leave details to the reader. 
Let $M$ be a nonparametric hypersurface, which is the graph of a $C^{2}$ function $u: \Omega \rightarrow \mathbb{R}$, where $\Omega \subset \mathbb{R}^{2 n+1}$ is an open bounded set, namely, $M=\left\{s=u\left(x_{1}, y_{1}, \ldots, x_{n}, y_{n}, t\right)\right\}$. In this case the coefficients $A_{\ell \bar{p}}\left(D u, D^{2} u\right)$ of the Levi form $L(u)$ are quasilinear partial differential operators whose real and imaginary parts are given by

$$
\begin{aligned}
\operatorname{Re}\left(A_{\ell \bar{p}}\left(D u, D^{2} u\right)\right)= & \left(\partial_{x_{\ell} x_{p}} u+\partial_{y_{\ell} y_{p}} u+a_{\ell} \partial_{x_{p} t} u+a_{p} \partial_{x_{\ell} t} u\right. \\
& \left.+b_{\ell} \partial_{y_{p} t} u+b_{p} \partial_{y_{\ell} t} u+\left(a_{\ell} a_{p}+b_{\ell} b_{p}\right) \partial_{t}^{2} u\right), \\
\operatorname{Im}\left(A_{\ell \bar{p}}\left(D u, D^{2} u\right)\right)= & \left(\partial_{x_{\ell} y_{p}} u-\partial_{x_{p} y_{\ell}} u-a_{p} \partial_{y_{\ell} t} u+a_{\ell} \partial_{y_{p} t} u\right. \\
& \left.+b_{p} \partial_{x_{\ell} t} u-b_{\ell} \partial_{x_{p} t} u+\left(b_{p} a_{\ell}-b_{\ell} a_{p}\right) \partial_{t}^{2} u\right)
\end{aligned}
$$

where

$$
a_{\ell}=a_{\ell}(D u)=\frac{\partial_{y_{\ell}} u-\partial_{x_{\ell}} u \partial_{t} u}{1+\left(\partial_{t} u\right)^{2}}, \quad b_{\ell}=b_{\ell}(D u)=\frac{-\partial_{x_{\ell}} u-\partial_{y_{\ell}} u \partial_{t} u}{1+\left(\partial_{t} u\right)^{2}}
$$

In particular for every $\ell=1, \ldots, n$, the diagonal coefficient $A_{\ell \bar{\ell}}\left(D u, D^{2} u\right)$ is a degenerate elliptic second order operator, whose characteristic form

$$
\xi=\left(\xi_{1}, \ldots, \xi_{2 n+1}\right) \longrightarrow\left(\xi_{2 l-1}+a_{\ell} \xi_{2 n+1}\right)^{2}+\left(\xi_{2 l}+b_{\ell} \xi_{2 n+1}\right)^{2},
$$

is nonnegative definite for every $\xi \in \mathbb{R}^{2 n+1}$, but has $2 n-1$ eigenvalues identically zero. In accordance with the notations of the Introduction, we define the Levi Monge-Ampère operator as

$$
\operatorname{LMA}(u)=\left(1+u_{t}^{2}\right) \operatorname{det}\left(A_{\ell, \bar{p}}\left(D u, D^{2} u\right)\right) .
$$

Definition 2.2. We say that a function $u \in C^{2}(\bar{\Omega})$ is Levi convex (strictly Levi convex) at $\xi_{0} \in \Omega$ if $L(u)\left(\xi_{0}\right) \geqslant 0$ (>0) and Levi convex (strictly Levi convex) in $\Omega$ if $L(u)(\xi) \geqslant 0$ (>0) for every $\xi \in \Omega$.

Remark 2.1. The following conditions are equivalent (see [20]):

(1) $u$ is Levi convex in $\Omega$,

(2) the matrix $A\left(D u, D^{2} u\right)=\left(A_{\ell, \bar{p}}\left(D u, D^{2} u\right)\right)_{\ell, p=1, \ldots, n}$ is nonnegative definite in $\Omega$,

(3) the epigraph of $u$ is pseudoconvex.

In [20] it has been proved that if $u \in C^{2}(\bar{\Omega})$ is convex in the classical sense, then $u$ is Levi convex. In particular, if $D^{2} u \geqslant 0$, then $A\left(D u, D^{2} u\right) \geqslant 0$. The converse obviously is not true.

For any $O \subseteq \mathbb{R}^{m}$, we denote by $\operatorname{USC}(O)$ the set of upper semicontinuous functions in $O$ and by $\operatorname{LSC}(O)$ the set of lower semicontinuous functions in $O$.

Definition 2.2 can be generalized to upper semicontinuous functions as follows (see also [23]).

Definition 2.3. We say that a function $u \in \operatorname{USC}(\bar{\Omega})$ is Levi convex (strictly Levi convex) in a viscosity sense at $\xi_{0} \in \Omega$ if for all $\phi \in C^{2}(\bar{\Omega})$ and for all local maximum $\xi_{0}$ of $u-\phi$ we have $L(\phi)\left(\xi_{0}\right) \geqslant 0(>0)$ and Levi convex (strictly Levi convex) in $\Omega$ if $L(\phi)(\xi) \geqslant 0(>0)$.

Now we give the definition of viscosity subsolution and supersolution to the following equation

$$
\operatorname{det} A\left(D u, D^{2} u\right)=k(\xi, u) f(D u) \quad \text { in } \Omega,
$$

where $k: \bar{\Omega} \times \mathbb{R} \rightarrow[0,+\infty)$ is a given continuous function and

$$
f(D u)=2^{n} \frac{\left(1+|D u|^{2}\right)^{(n+2) / 2}}{\left(1+\left(\partial_{t} u\right)^{2}\right)} .
$$


Our definition extends the one given by Ishii and Lions in [16] in the case of the classical Monge-Ampère equation, and it is analogous to that introduced by Slodkowski and Tomassini in [23] for the Dirichlet problem (1.2).

Definition 2.4. We say that $u \in \operatorname{USC}(\bar{\Omega})$ (resp. $v \in \operatorname{LSC}(\bar{\Omega})$ ) is a viscosity subsolution (resp. supersolution) of (2.4) if for all $\phi \in C^{2}(\bar{\Omega})$ the following holds: at each local maximum $\xi_{0}$ (resp. local minimum ) point of $u-\phi$ $(v-\phi)$ then

$$
\operatorname{det} A\left(D \phi, D^{2} \phi\right)\left(\xi_{0}\right) \geqslant k\left(\xi_{0}, u\left(\xi_{0}\right)\right) f\left(D \phi\left(\xi_{0}\right)\right)
$$

and

$$
L(\phi)\left(\xi_{0}\right) \geqslant 0
$$

(resp. either $L(\phi)\left(\xi_{0}\right)$ is not semidefinite positive or

$$
\operatorname{det} A\left(D \phi, D^{2} \phi\right)\left(\xi_{0}\right) \leqslant k\left(\xi_{0}, u\left(\xi_{0}\right)\right) f\left(D \phi\left(\xi_{0}\right)\right)
$$

and

$$
\left.L(\phi)\left(\xi_{0}\right) \geqslant 0\right)
$$

We recall that for all $A \in \mathcal{S}^{n}$ (the set of $n \times n$ symmetric matrices) the following matrix identity holds:

$$
(\operatorname{det} A)^{1 / n}= \begin{cases}\inf \left\{\operatorname{Tr}(A B): B \in \mathcal{S}^{n}, B \geqslant 0, \operatorname{det} B=n^{-n}\right\}, & \text { if } A \geqslant 0, \\ -\infty, & \text { otherwise }\end{cases}
$$

In view of the identity (2.5), we give another viscosity formulation of the Dirichlet problem (1.2). To this purpose we consider the operator $F: \Omega \times \mathbb{R} \times \mathbb{R}^{N} \times \mathcal{S}^{N} \rightarrow \mathbb{R}$ with $N=2 n+1$, defined by

$$
F(\xi, u, p, X):= \begin{cases}k^{1 / n}(\xi, u) f^{1 / n}(p)-(\operatorname{det} A(p, X))^{1 / n}, & \text { if } A(p, X) \geqslant 0, \\ +\infty, & \text { otherwise }\end{cases}
$$

and the Dirichlet problem

$$
\begin{cases}F\left(\xi, u, D u, D^{2} u\right)=0 & \text { in } \Omega, \\ u(\xi)=\varphi(\xi), & \text { in } \partial \Omega,\end{cases}
$$

where $\varphi \in C(\partial \Omega)$ the solution $u$ is a scalar function and $D u$ and $D^{2} u$ denote respectively its gradient and Hessian matrix.

We set $F^{*}$ and $F_{*}$ the usc and lsc envelope of $F$ respectively.

Definition 2.5. A function $u \in \operatorname{USC}(\bar{\Omega})$ (resp. $v \in \operatorname{LSC}(\bar{\Omega})$ ) is said to be a viscosity subsolution (resp. supersolution) of (DP) in a generalized sense iff the following property holds:

for all $\phi \in C^{2}(\bar{\Omega})$, at each maximum point $\xi_{0} \in \bar{\Omega}$ of $u-\phi$ we have

$$
\begin{aligned}
& F_{*}\left(\xi_{0}, u\left(\xi_{0}\right), D \phi\left(\xi_{0}\right), D^{2} \phi\left(\xi_{0}\right)\right) \leqslant 0 \quad \text { if } \xi_{0} \in \Omega, \\
& \min \left(F_{*}\left(\xi_{0}, u\left(\xi_{0}\right), D \phi\left(\xi_{0}\right), D^{2} \phi\left(\xi_{0}\right)\right), u\left(\xi_{0}\right)-\varphi\left(\xi_{0}\right)\right) \leqslant 0 \quad \text { if } \xi_{0} \in \partial \Omega,
\end{aligned}
$$

(resp. for all $\phi \in C^{2}(\bar{\Omega})$, at each minimum point $\xi_{0} \in \bar{\Omega}$ of $u-\phi$ we have

$$
\begin{aligned}
& F^{*}\left(\xi_{0}, u\left(\xi_{0}\right), D \phi\left(\xi_{0}\right), D^{2} \phi\left(\xi_{0}\right)\right) \geqslant 0 \quad \text { if } \xi_{0} \in \Omega, \\
& \max \left(F^{*}\left(\xi_{0}, u\left(\xi_{0}\right), D \phi\left(\xi_{0}\right), D^{2} \phi\left(\xi_{0}\right)\right), u\left(\xi_{0}\right)-\varphi\left(\xi_{0}\right)\right) \geqslant 0 \quad \text { if } \xi_{0} \in \partial \Omega .
\end{aligned}
$$

Proposition 2.2. Every solution u of (2.4) at $\xi_{0} \in \Omega$ in the sense of Definition 2.4 is a solution of $F=0$ in the sense of Definition 2.5. 
Proof. We show that every solution of $F=0$ in the sense of Definition 2.5 is a solution of (2.4) in the sense of Definition 2.4, the other implication being evident. Let $u$ be a generalized solution of (DP) and let $\phi \in C^{2}(\Omega)$ be such that $u-\phi$ has a maximum at $\xi_{0} \in \Omega$, then the inequality $F_{*}\left(\xi_{0}, u\left(\xi_{0}\right), D \phi\left(\xi_{0}\right), D^{2} \phi\left(\xi_{0}\right)\right) \leqslant 0$ implies that $L(\phi)\left(\xi_{0}\right) \geqslant 0$. Thus we have

$$
\operatorname{det} A\left(D \phi, D^{2} \phi\right)\left(\xi_{0}\right) \geqslant k\left(\xi_{0}, u\left(\xi_{0}\right)\right) f\left(D \phi\left(\xi_{0}\right)\right)
$$

and

$$
L(\phi)\left(\xi_{0}\right) \geqslant 0 .
$$

Now suppose that $u-\phi$ has a minimum at $\xi_{0} \in \Omega$ and that $F^{*}\left(\xi_{0}, u\left(\xi_{0}\right), D \phi\left(\xi_{0}\right), D^{2} \phi\left(\xi_{0}\right)\right) \geqslant 0$. We distinguish the following two cases:

(1) $L(\phi)\left(\xi_{0}\right) \geqslant 0$ and $L(\phi)\left(\xi_{0}\right)$ has at least one null eigenvalue. In this case we have

$$
0=\operatorname{det} A\left(D \phi, D^{2} \phi\right)\left(\xi_{0}\right) \leqslant k\left(\xi_{0}, u\left(\xi_{0}\right)\right) f\left(D \phi\left(\xi_{0}\right)\right) .
$$

(2) $L(\phi)\left(\xi_{0}\right)>0$, then there is a ball $B\left(\xi_{0}, r\right), r>0$, such that $L(\phi)(y)>0$ for all $y \in B\left(\xi_{0}, r\right)$. It follows that

$$
F^{*}\left(\xi_{0}, u\left(\xi_{0}\right), D \phi\left(\xi_{0}\right), D^{2} \phi\left(\xi_{0}\right)\right)=F\left(\xi_{0}, u\left(\xi_{0}\right), D \phi\left(\xi_{0}\right), D^{2} \phi\left(\xi_{0}\right)\right)
$$

and the inequality $F^{*}\left(\xi_{0}, u\left(\xi_{0}\right), D \phi\left(\xi_{0}\right), D^{2} \phi\left(\xi_{0}\right)\right) \geqslant 0$ implies

$$
\operatorname{det} A\left(D \phi, D^{2} \phi\right)\left(\xi_{0}\right) \leqslant k\left(\xi_{0}, u\left(\xi_{0}\right)\right) f\left(D \phi\left(\xi_{0}\right)\right)
$$

and

$$
L(\phi)\left(\xi_{0}\right) \geqslant 0 .
$$

Hence we can conclude.

In the sequel when we talk about sub- and supersolutions of (DP), we will always mean in a viscosity sense.

We explicitly remark that subsolutions of (DP) are Levi convex in a viscosity sense. Moreover, standard calculations show that if $u \in C^{2}(\Omega) \cap C(\bar{\Omega})$ is Levi convex, then $u$ is a classical solution of (DP) iff $u$ is a viscosity solution of (DP) (see [23]).

\section{Loss of boundary conditions}

Let $\Omega$ be a bounded open set of $\mathbb{R}^{N}, N=2 n+1$, with $C^{2}$ boundary. Denote by $d$ a smooth function agreeing in a neighborhood $\mathcal{W}$ of $\partial \Omega$ with the signed distance function to $\partial \Omega$ which is positive in $\Omega$ and negative in $\mathbb{R} \backslash \bar{\Omega}$ and we denote by $n(\xi):=-D d(\xi)$ for all $\xi \in \mathcal{W}$. If $\xi \in \partial \Omega, n(\xi)$ is just the unit outward normal to $\partial \Omega$ at $\xi$.

In this section we analyze the loss of boundary conditions for the Dirichlet problem (DP) where $F$ is given by (2.6). The question of loss of boundary conditions have been addressed by the first author in [10] for general fully nonlinear second order degenerate elliptic and parabolic equations. As it is well known this fact may depend on various aspects, such as the geometry of the domains, the structural properties of the operator appearing in the equation and the value of the boundary data (see e.g. the example in [5]). Here we are going to test on the operator (2.6) the conditions which have been found in [10] implying that the Dirichlet boundary conditions are assumed continuously by the solutions of (DP). To this end we introduce the following subsets of the boundary $\partial \Omega$ : we denote by $\Sigma_{-}$the set of the points $\xi \in \partial \Omega$ such that, for all $R>0$ either

$$
\liminf _{\substack{w \rightarrow \xi \\ \alpha \downarrow 0}}\left\{F\left(w,-R, \frac{-n(w)+o_{\alpha}(1)}{\alpha},-\frac{1}{\alpha^{2}} n(w) \otimes n(w)+\frac{o_{\alpha}(1)}{\alpha^{2}}\right)\right\}>0
$$


or

$$
\liminf _{\substack{w \rightarrow \xi \\ \alpha \downarrow 0}}\left\{F\left(w,-R, \frac{-n(w)+o_{\alpha}(1)}{\alpha}, \frac{1}{\alpha} D^{2} d(w)+\frac{o_{\alpha}(1)}{\alpha}\right)\right\}>0
$$

and we denote by $\Sigma_{+}$the set of the points $\xi \in \partial \Omega$ such that, for all $R>0$

$$
\limsup _{\substack{w \rightarrow \xi \\ \alpha \downarrow 0}}\left\{F\left(w, R, \frac{n(w)+o_{\alpha}(1)}{\alpha}, \frac{1}{\alpha^{2}} n(w) \otimes n(w)+\frac{o_{\alpha}(1)}{\alpha^{2}}\right)\right\}<0
$$

or

$$
\limsup _{\substack{w \rightarrow \xi \\ \alpha \downarrow 0}}\left\{F\left(w, R, \frac{n(w)+o_{\alpha}(1)}{\alpha},-\frac{1}{\alpha} D^{2} d(w)+\frac{o_{\alpha}(1)}{\alpha}\right)\right\}<0,
$$

where $o_{\alpha}(1) \rightarrow 0$ as $\alpha \downarrow 0$ and $p \otimes p$ is the matrix $\left(p_{i} p_{j}\right)_{i, j=1}^{N}$, for all $p=\left(p_{1}, \ldots, p_{N}\right)$. Finally we set

$$
\Sigma:=\partial \Omega \backslash\left(\Sigma_{-} \cup \Sigma_{+}\right) .
$$

We premise some comments on the sets $\Sigma_{ \pm}$. In Section 4 of [10], it is proved that there cannot be loss of boundary conditions respectively for the sub- and supersolutions of (DP), namely for every $\xi \in \Sigma_{-}$(resp. $\Sigma_{+}$) and any subsolutions $u$ (resp. supersolutions $v$ ) we have $u(\xi) \leqslant \varphi(\xi)(v(x) \geqslant \varphi(\xi))$.

We will show that condition (H3) on $\partial \Omega$ is enough to guarantee that $\Sigma=\emptyset$.

Now we are going to test the conditions (3.1)-(3.3) and (3.4) in the case of the operator (2.6). We first note that if $u$ is a defining function for $\Omega$ then the Levi curvature (1.1) of $M=\left\{(\xi, s) \in \mathbb{R}^{2 n+2}: u(x, y, t)-s=0\right\}$ can be represented as follows

$$
k_{M}(\xi, u)=-h(D u) \operatorname{det} B\left(D u, D^{2} u\right)
$$

with $h(D u):=2^{n+2}\left(1+|D u|^{2}\right)^{-((n+2) / 2)}$ and

$$
B\left(D u, D^{2} u\right)=\left(\begin{array}{cccc}
0 & \partial_{\overline{1}} u & \cdots & \frac{\partial_{t} u-\mathrm{i}}{2} \\
\partial_{1} u & \partial_{1 \overline{1}} u & \cdots & \frac{\partial_{1 t} u}{2} \\
\vdots & \vdots & \ddots & \vdots \\
\frac{\partial_{t} u+\mathrm{i}}{2} & \frac{\partial_{t \overline{1}} u}{2} & \cdots & \frac{\partial_{t t} u}{4}
\end{array}\right) .
$$

Define

$$
k_{M}^{\infty}(\xi):=\lim _{\eta \rightarrow \infty} k_{M}(\xi, \eta s) .
$$

Then

$$
k_{M}^{\infty}(\xi)=-2^{n}|D u|^{-(n+2)} \operatorname{det} B_{\infty}\left(D u, D^{2} u\right),
$$

with

$$
B_{\infty}\left(D u, D^{2} u\right):=\left(\begin{array}{cccc}
0 & \partial_{\overline{1}} u & \cdots & \partial_{t} u \\
\partial_{1} u & \partial_{1 \overline{1}} u & \cdots & \partial_{1 t} u \\
\vdots & \vdots & \ddots & \vdots \\
\partial_{t} u & \partial_{\overline{1} \overline{1}} u & \cdots & \partial_{t t} u
\end{array}\right)
$$


and $k_{M}^{\infty}(\xi)$ is exactly the Levi curvature $k_{\partial \Omega \times i \mathbb{R}}$ of the cylinder $\partial \Omega \times \mathrm{i} \mathbb{R}=\{(\xi, s): u(\xi)=0\}$. By algebraic computations one can rewrite $k_{M}$ also as follows (see e.g [20])

$$
k_{M}(\xi, u)=\frac{1}{2^{n}} \frac{\left(1+u_{t}^{2}\right)}{\left(1+|D u|^{2}\right)^{(n+2) / 2}} \operatorname{det} A\left(D u, D^{2} u\right),
$$

where $A(p, X)$ is the $n \times n$ Hermitian matrix defined in (2.1).

We list below some facts on the matrix $A$ that will be useful to check (3.1)-(3.4) on a boundary point. First we have

$$
A(\eta p, \eta X)=\frac{\eta}{\left(\eta^{-2}+p_{N}^{2}\right)^{2}} A^{\prime}(p, X, \eta)
$$

where the coefficients of $A^{\prime}(p, X, \eta)$ are given by

$$
\begin{aligned}
\operatorname{Re}\left(A_{\ell \bar{p}}^{\prime}\left(D u, D^{2} u, \eta\right)\right)= & \left(\eta^{-2}+u_{t}^{2}\right)^{2}\left(\partial_{x_{\ell} x_{p}} u+\partial_{y_{\ell} y_{p}} u\right)+\left(\eta^{-2}+u_{t}^{2}\right)\left(a_{\ell}^{\prime} \partial_{x_{p} t} u+a_{p}^{\prime} \partial_{x_{\ell} t} u\right. \\
& \left.+b_{\ell}^{\prime} \partial_{y_{p} t} u+b_{p}^{\prime} \partial_{y_{\ell} t} u\right)+\left(a_{\ell}^{\prime} a_{p}^{\prime}+b_{\ell}^{\prime} b_{p}^{\prime}\right) \partial_{t}^{2} u, \\
\operatorname{Im}\left(A_{\ell \bar{p}}^{\prime}\left(D u, D^{2} u, \eta\right)\right)= & \left(\eta^{-2}+u_{t}^{2}\right)^{2}\left(\partial_{x_{\ell} y_{p}} u-\partial_{x_{p} y_{\ell}} u\right)+\left(\eta^{-2}+u_{t}^{2}\right)\left(-a_{p}^{\prime} \partial_{y_{\ell} t} u+a_{\ell}^{\prime} \partial_{y_{p} t} u\right. \\
& \left.+b_{p}^{\prime} \partial_{x_{\ell} t} u-b_{\ell}^{\prime} \partial_{x_{p} t} u\right)+\left(b_{p}^{\prime} a_{\ell}^{\prime}-b_{\ell}^{\prime} a_{p}^{\prime}\right) \partial_{t}^{2} u
\end{aligned}
$$

and

$$
a_{\ell}^{\prime}=a_{\ell}^{\prime}(D u, \eta)=\eta^{-1} \partial_{y_{\ell}} u-\partial_{x_{\ell}} u \partial_{t} u, \quad b_{\ell}^{\prime}=b_{\ell}^{\prime}(D u, \eta)=-\eta^{-1} \partial_{x_{\ell}} u-\partial_{y_{\ell}} u \partial_{t} u .
$$

Moreover $A^{\prime}(p, X, \eta)$ converges to $A_{\infty}(p, X)$ as $\eta \rightarrow \infty$ locally uniformly in $(p, X)$, where the real part and the imaginary part of $\left(A_{\infty}\right)_{\ell} \bar{p}\left(D u, D^{2} u\right)$ are given by

$$
\begin{aligned}
\operatorname{Re}\left(\left(A_{\infty}\right)_{\ell \bar{p}}\left(D u, D^{2} u\right)\right)= & \left(u_{t}\right)^{4}\left(\partial_{x_{\ell} x_{p}} u+\partial_{y_{\ell} y_{p}} u\right)+u_{t}^{2}\left(a_{\ell}^{\infty} \partial_{x_{p} t} u+a_{p}^{\infty} \partial_{x_{\ell} t} u\right. \\
& \left.+b_{\ell}^{\infty} \partial_{y_{p} t} u+b_{p}^{\infty} \partial_{y_{\ell} t} u\right)+\left(a_{\ell}^{\infty} a_{p}^{\infty}+b_{\ell}^{\infty} b_{p}^{\infty}\right) \partial_{t}^{2} u, \\
\operatorname{Im}\left(\left(A_{\infty}\right)_{\ell \bar{p}}\left(D u, D^{2} u\right)\right)= & \left(u_{t}\right)^{4}\left(\partial_{x_{\ell} y_{p}} u-\partial_{x_{p} y_{\ell}} u\right)+u_{t}^{2}\left(-a_{p}^{\infty} \partial_{y_{\ell} t} u+a_{\ell}^{\infty} \partial_{y_{p} t} u\right. \\
& \left.+b_{p}^{\infty} \partial_{x_{\ell} t} u-b_{\ell}^{\infty} \partial_{x_{p} t} u\right)+\left(b_{p}^{\infty} a_{\ell}^{\infty}-b_{\ell}^{\infty} a_{p}^{\infty}\right) \partial_{t}^{2} u
\end{aligned}
$$

with

$$
a_{\ell}^{\infty}=a_{\ell}^{\infty}(D u)=-\partial_{x_{\ell}} u \partial_{t} u, \quad b_{\ell}^{\infty}=b_{\ell}^{\infty}(D u)=-\partial_{y_{\ell}} u \partial_{t} u .
$$

Next we start analyzing the two conditions (3.1), (3.3). Standard computations show that $\operatorname{det} B_{\infty}(n, n \otimes n)=$ $\operatorname{det} B_{\infty}(-n,-n \otimes n)=0$.

Now we take $\xi_{0} \in \partial \Omega$ and we distinguish two cases.

Case 1: for all $\alpha>0$ small and for all $w$ close to $\xi_{0}$ the matrix $A\left(\frac{-n(w)+o_{\alpha}(1)}{\alpha},-\frac{1}{\alpha^{2}} n(w) \otimes n(w)+\frac{o_{\alpha}(1)}{\alpha^{2}}\right)$ is not semidefinite positive. In this case we trivially have

$$
\liminf _{\substack{w \rightarrow \xi \\ \alpha \downarrow 0}}\left\{F\left(w, R, \frac{-n(w)+o_{\alpha}(1)}{\alpha},-\frac{1}{\alpha^{2}} n(w) \otimes n(w)+\frac{o_{\alpha}(1)}{\alpha^{2}}\right)\right\} \geqslant 0 .
$$

Case 2: there are subsequences $\alpha_{n} \rightarrow 0$ and $w_{n} \rightarrow \xi_{0}$ (that we continue to denote by $\alpha$ and $w$ ) such that the matrix $A\left(\frac{-n(w)+o_{\alpha}(1)}{\alpha},-\frac{1}{\alpha^{2}} n(w) \otimes n(w)+\frac{o_{\alpha}(1)}{\alpha^{2}}\right)$ is semidefinite positive. In this case the following estimate holds.

$$
\begin{aligned}
& F\left(w,-R, \frac{-n(w)+o_{\alpha}(1)}{\alpha},-\frac{1}{\alpha^{2}} n(w) \otimes n(w)+\frac{o_{\alpha}(1)}{\alpha^{2}}\right)=f^{1 / n}\left(\frac{-n(w)+o_{\alpha}(1)}{\alpha}\right)\left\{k^{1 / n}(w,-R)\right. \\
& \left.\quad-\left[\operatorname{det} A\left(\frac{-n(w)+o_{\alpha}(1)}{\alpha},-\frac{1}{\alpha^{2}} n(w) \otimes n(w)+\frac{o_{\alpha}(1)}{\alpha^{2}}\right) f^{-1}\left(\frac{-n(w)+o_{\alpha}(1)}{\alpha}\right)\right]^{1 / n}\right\} .
\end{aligned}
$$


By using the identities (3.7), (3.8) and the fact that $\operatorname{det} B_{\infty}(-n,-n \otimes n)=0$, we get

$$
\begin{aligned}
& \liminf _{\substack{w \rightarrow \xi_{0} \\
\alpha \downarrow 0}}-\left[\operatorname{det} A\left(\frac{-n(w)+o_{\alpha}(1)}{\alpha},-\frac{1}{\alpha^{2}} n(w) \otimes n(w)+\frac{o_{\alpha}(1)}{\alpha^{2}}\right) f^{-1}\left(\frac{-n(w)+o_{\alpha}(1)}{\alpha}\right)\right]^{1 / n} \\
& \quad+k^{1 / n}(w,-R) \\
& \quad \geqslant \liminf _{\substack{\alpha \rightarrow \xi_{0} \\
\alpha \downarrow 0}}\left[-2\left(-\operatorname{det}\left(B_{\infty}(-n(w),-n(w) \otimes n(w))\right)^{1 / n}+k^{1 / n}(w,-R)\right]\right. \\
& \quad=k^{1 / n}\left(\xi_{0},-R\right) \geqslant 0 .
\end{aligned}
$$

Thus since $f^{1 / n} \geqslant 0$ we finally obtain

$$
\begin{aligned}
& \liminf _{\substack{w \rightarrow \xi_{0} \\
\alpha \downarrow 0}}\left\{F\left(w,-R, \frac{-n(w)+o_{\alpha}(1)}{\alpha},-\frac{1}{\alpha^{2}} n(w) \otimes n(w)+\frac{o_{\alpha}(1)}{\alpha^{2}}\right)\right\} \\
& \quad \geqslant \liminf _{\substack{w \rightarrow \xi_{0} \\
\alpha \downarrow 0}} f^{1 / n}\left(\frac{-n(w)+o_{\alpha}(1)}{\alpha}\right) k^{1 / n}\left(\xi_{0},-R\right) \geqslant 0 .
\end{aligned}
$$

In a similar way one sees that

$$
\limsup _{\substack{w \rightarrow \xi_{0} \\ \alpha \downarrow 0}}\left\{F\left(w, R, \frac{n(w)+o_{\alpha}(1)}{\alpha}, \frac{1}{\alpha^{2}} n(w) \otimes n(w)+\frac{o_{\alpha}(1)}{\alpha^{2}}\right)\right\} \geqslant 0 .
$$

Thus the conditions (3.1) and (3.3) are not satisfied.

This implies that we have to impose some suitable conditions on the Levi curvature of the domain in order that both conditions (3.2) and (3.4) hold.

To this end we assume that $\Omega$ satisfies (H3). Then since $-d$ is a defining function of $\Omega$ the following two conditions holds for every $s \in \mathbb{R}$ and $\xi_{0} \in \partial \Omega$ :

$$
A_{\infty}\left(n\left(\xi_{0}\right),-D^{2} d\left(\xi_{0}\right)\right)>0
$$

and

$$
2\left(-\operatorname{det}\left(B_{\infty}\left(n\left(\xi_{0}\right),-D^{2} d\left(\xi_{0}\right)\right)\right)\right)^{1 / n}>k^{1 / n}\left(\xi_{0}, s\right) .
$$

Proposition 3.1. Assume (H3) then both the conditions (3.2) and (3.4) are satisfied.

Proof. We first notice that $A_{\infty}\left(-n\left(\xi_{0}\right), D^{2} d\left(\xi_{0}\right)\right)=-A_{\infty}\left(n\left(\xi_{0}\right),-D^{2} d\left(\xi_{0}\right)\right)$ is not semidefinite positive.

We claim that the matrix

$$
A\left(\frac{-n(w)+o_{\alpha}(1)}{\alpha}, \frac{1}{\alpha} D^{2} d(w)+\frac{o_{\alpha}(1)}{\alpha}\right)
$$

is not semidefinite positive for $\alpha \rightarrow 0$ and $w \rightarrow \xi_{0}$ as well.

Indeed since $A_{\infty}\left(-n\left(\xi_{0}\right), D^{2} d\left(\xi_{0}\right)\right)$ is not semidefinite positive, there is at least one eigenvalue which is strictly negative. Now from (3.9) it follows that

$$
\begin{aligned}
A & \left(\frac{-n(w)+o_{\alpha}(1)}{\alpha}, \frac{1}{\alpha} D^{2} d(w)+\frac{o_{\alpha}(1)}{\alpha}\right) \\
& =\frac{\alpha^{-1}}{\left(\alpha^{2}+\left(d_{t}+o_{\alpha}(1)^{2}\right)^{2}\right.} A^{\prime}\left(D d+o_{\alpha}(1), D^{2} d+o_{\alpha}(1), \alpha^{-1}\right) .
\end{aligned}
$$


Since

$$
A^{\prime}\left(D d(w)+o_{\alpha}(1), D^{2} d(w)+o_{\alpha}(1), \alpha^{-1}\right) \rightarrow A_{\infty}\left(D d\left(\xi_{0}\right), D^{2} d\left(\xi_{0}\right)\right)
$$

as $\alpha \rightarrow 0$ and $w \rightarrow \xi_{0}$, one can see that there are $r>0$ and $\alpha_{0}>0$ such that for all $0<\alpha \leqslant \alpha_{0}$ and for all $w \in B\left(\xi_{0}, r\right)$ the matrix $A^{\prime}\left(D d(w)+o_{\alpha}(1), D^{2} d(w)+o_{\alpha}(1), \alpha^{-1}\right)$ is not semidefinite positive. Thus from (3.14) it follows that the matrix

$$
A\left(\frac{-n(w)+o_{\alpha}(1)}{\alpha}, \frac{1}{\alpha} D^{2} d(w)+\frac{o_{\alpha}(1)}{\alpha}\right)
$$

is not semidefinite positive too and we prove the claim.

Hence we have

$$
\liminf _{\substack{w \rightarrow \xi_{0} \\ \alpha \downarrow 0}}\left\{F\left(w,-R, \frac{-n(w)+o_{\alpha}(1)}{\alpha}, \frac{1}{\alpha} D^{2} d(w)+\frac{o_{\alpha}(1)}{\alpha}\right)\right\}=+\infty
$$

and (3.2) holds.

On the other hand if $A_{\infty}\left(n\left(\xi_{0}\right), D^{2} d\left(\xi_{0}\right)\right)$ is definite positive then the matrix

$$
A\left(\frac{n(w)+o_{\alpha}(1)}{\alpha},-\frac{1}{\alpha} D^{2} d(w)+\frac{o_{\alpha}(1)}{\alpha}\right)
$$

is definite positive as $\alpha \rightarrow 0$ and $w \rightarrow \xi_{0}$. To show this fact one argues exactly as above. Thus we have

$$
\begin{aligned}
& \limsup _{\substack{w \rightarrow \xi_{0} \\
\alpha \downarrow 0}}\left\{F\left(w, R, \frac{n(w)+o_{\alpha}(1)}{\alpha},-\frac{1}{\alpha} D^{2} d(w)+\frac{o_{\alpha}(1)}{\alpha}\right)\right\}=\limsup _{\substack{w \rightarrow \xi_{0} \\
\alpha \downarrow 0}} f^{1 / n}\left(\frac{n(w)+o_{\alpha}(1)}{\alpha}\right), \\
& \underset{\substack{w \rightarrow \xi_{0} \\
\lim \sup _{0}}}{ }\left\{-\left(\operatorname{det}\left(A\left(\frac{n(w)+o_{\alpha}(1)}{\alpha},-\frac{1}{\alpha} D^{2} d(w)+\frac{o_{\alpha}(1)}{\alpha}\right) f^{-1}\left(\frac{n(w)+o_{\alpha}(1)}{\alpha}\right)\right)^{1 / n}+k^{1 / n}(w, R)\right\}\right. \\
& \quad \leqslant \limsup _{\substack{w \rightarrow \xi_{0} \\
\alpha \downarrow 0}} f^{1 / n}\left(\frac{-n(w)+o_{\alpha}(1)}{\alpha}\right) \cdot\left\{-2\left(-\operatorname{det}\left(B_{\infty}\left(n\left(\xi_{0}\right),-D^{2} d\left(\xi_{0}\right)\right)\right)\right)^{1 / n}+k^{1 / n}\left(\xi_{0}, R\right)\right\}<0,
\end{aligned}
$$

where the last inequality follows by combining (3.13) and the fact that $f^{1 / n} \geqslant 2$. Therefore (3.4) is satisfied and we conclude.

\section{Comparison principles and existence results}

In this section we provide two comparison principles between viscosity semicontinuous subsolutions and supersolutions of (DP) under the hypothesis (H3), which guarantees that the boundary data is assumed continuously.

As a by-product of the these comparison results and the Perron's method we get the existence of a unique continuous viscosity solution of (DP).

The first comparison result of this section is the following theorem, which holds under the assumption that the function $k$ is strictly increasing with respect to $u$. The proof of this result is standard and we provide it for the reader's convenience.

Theorem 4.1. Assume (H1)-(H3). Let $u \in \operatorname{USC}(\bar{\Omega})$ and $v \in \operatorname{LSC}(\bar{\Omega})$ be respectively a bounded viscosity subsolution and supersolution of (DP). Then $u \leqslant v$ in $\bar{\Omega}$. 
Proof. We suppose by contradiction that $\max _{\bar{\Omega}}(u-v)=M>0$. By (H3) such maximum is achieved at an interior point $\xi_{0}$. For all $\varepsilon>0$ we consider the auxiliary function

$$
\Phi_{\varepsilon}(\xi, \zeta)=u(\xi)-v(\zeta)-\frac{|\xi-\zeta|^{2}}{\varepsilon^{2}} .
$$

Let $\left(\xi_{\varepsilon}, \zeta_{\varepsilon}\right)$ be a maximum of $\Phi_{\varepsilon}$ in $\bar{\Omega} \times \bar{\Omega}$. By standard arguments we get, up to subsequences, that $\xi_{\varepsilon}, \zeta_{\varepsilon} \rightarrow \tilde{\xi} \in$ $\bar{\Omega}$, and

$$
\begin{aligned}
& \frac{\left|\xi_{\varepsilon}-\zeta_{\varepsilon}\right|^{2}}{\varepsilon^{2}}=o_{\varepsilon}(1) \quad \text { as } \varepsilon \rightarrow 0, \\
& u\left(\xi_{\varepsilon}\right)-v\left(\zeta_{\varepsilon}\right) \rightarrow u(\tilde{\xi})-v(\tilde{\xi})=M, \\
& u\left(\xi_{\varepsilon}\right) \rightarrow u(\tilde{\xi}), v\left(\zeta_{\varepsilon}\right) \rightarrow v(\tilde{\xi}) .
\end{aligned}
$$

We observe that since $u \leqslant v$ on $\partial \Omega$ we have $\tilde{\xi} \in \Omega$, thus for $\varepsilon$ small enough $\xi_{\varepsilon}, \zeta_{\varepsilon} \in \Omega$ as well. Hence the equation holds for both $u$ and $v$ respectively at $\xi_{\varepsilon}$ and $\zeta_{\varepsilon}$.

Set $\phi(\xi, \zeta)=|\xi-\zeta|^{2} / \varepsilon^{2}$. For all $\alpha>0$ there exist $X, Y \in \mathcal{S}(N)$ such that, if $p_{\varepsilon}:=2\left(\xi_{\varepsilon}-\zeta_{\varepsilon}\right) / \varepsilon^{2}$, and $A=$ $D^{2} \phi\left(\xi_{\varepsilon}, \zeta_{\varepsilon}\right)$, we have

$$
\begin{aligned}
& \left(p_{\varepsilon}, X\right) \in \mathcal{J}^{2,+} u\left(\xi_{\varepsilon}\right), \quad\left(p_{\varepsilon}, Y\right) \in \mathcal{J}^{2,-} v\left(\zeta_{\varepsilon}\right), \\
& -\left(\frac{1}{\alpha}+\|A\|\right) \operatorname{Id} \leqslant\left(\begin{array}{cc}
X & 0 \\
0 & -Y
\end{array}\right) \leqslant A+\alpha A^{2}
\end{aligned}
$$

and

$$
F_{*}\left(\xi_{\varepsilon}, u\left(\xi_{\varepsilon}\right), p_{\varepsilon}, X\right) \leqslant 0 \quad \text { and } \quad F^{*}\left(\zeta_{\varepsilon}, v\left(\zeta_{\varepsilon}\right), p_{\varepsilon}, Y\right) \geqslant 0
$$

We note that (4.1) implies $X \leqslant Y$, thus $A\left(p_{\varepsilon}, X\right) \leqslant A\left(p_{\varepsilon}, Y\right)$ for all $\varepsilon$ as well. By subtracting the two inequalities in (4.2) and by using (H1) we get

$$
\ell_{R}(u(\tilde{\xi})-v(\tilde{\xi})) \leqslant o(1) \quad \text { as } \varepsilon \rightarrow 0
$$

and we obtain a contradiction by letting $\varepsilon \rightarrow 0$.

Next we are going to prove a comparison result by assuming the weaker condition (H5). When there is not a strict monotonicity with respect to $u$, one of the classical approaches from the theory of viscosity solutions, is to try to find a strict subsolution or supersolution either of the original equation or of a suitable approximation of it. Here we extend the techniques used in Ishii and Lions [16] for quasilinear equations.

To this purpose we need the following two lemmae.

Lemma 4.1. There is a function $\psi \in C^{2}(\bar{\Omega})$ such that

$$
\inf _{p \in \mathbb{R}^{2 n+1}}\left(\operatorname{det} A\left(p, D^{2} \psi\right)\right)^{1 / n}=v>0 .
$$

Proof. Let us take $\psi(x, y, t)=g\left(\left(\|x\|^{2}+\|y\|^{2}\right) / 2\right)$, with $g \in C^{2}(\mathbb{R})$ and $g^{\prime}, g^{\prime \prime}>0$. We note that for all $p \in \mathbb{R}^{N}$ and $X \in \mathcal{S}^{N}$ we have $A(p, Y)=\sigma(p) Y \bar{\sigma}^{\mathrm{T}}(p)$ where $\sigma$ is the $n \times N$ matrix given by

$$
\sigma(p)=\left(I_{n}, \quad-\mathrm{i} I_{n}, \quad a(p)-\mathrm{i} b(p)\right)
$$

$a(\cdot), b(\cdot)$ being defined in $(2.1)$. By using the above identity one can readily see that we have

$$
A\left(p, D^{2} \psi\right)=2 g^{\prime}\left(I_{n}+\frac{g^{\prime \prime}}{2 g^{\prime}}(x-\mathrm{i} y) \otimes(x+\mathrm{i} y)\right)
$$


and

$$
\left(\operatorname{det} A\left(p, D^{2} \psi\right)\right)^{1 / n} \geqslant 2 g^{\prime}\left(1+\frac{g^{\prime \prime}}{2 g^{\prime}}\left(\|x\|^{2}+\|y\|^{2}\right)\right)^{1 / n}=v .
$$

Lemma 4.2. If $u \in \operatorname{USC}(\bar{\Omega})$ is a bounded viscosity subsolution of $F=0$, then $u_{m}=u+\frac{1}{m} \psi$, with $\psi$ as in the previous lemma, is a strictly viscosity subsolution of

$$
F\left(\xi, u_{m}, D u_{m}-D \psi / m, D^{2} u_{m}\right)+f^{1 / n}\left(D u_{m}-D \psi / m\right)\left(k^{1 / n}\left(\xi, u_{m}-\psi / m\right)-k^{1 / n}\left(\xi, u_{m}\right)\right)=-\frac{v}{m} .
$$

Proof. We notice that for all $\phi \in C^{2}(\bar{\Omega}), \xi_{0}$ is a maximum point of $u_{m}-\phi$, iff $\xi_{0}$ is a maximum point of $u-(\phi-$ $\psi / m$ ). Thus, since $u$ is a viscosity subsolution of $F=0$, at each maximum point of $u_{m}-\phi$, we have

$$
F\left(\xi_{0}, u\left(\xi_{0}\right),(D \phi-D \psi / m)\left(\xi_{0}\right),\left(D^{2} \phi-D^{2} \psi / m\right)\left(\xi_{0}\right)\right) \leqslant 0 .
$$

Therefore, by the convexity of the function $A \mapsto-(\operatorname{det} A)^{1 / n}$ and by Lemma 4.1, we get

$$
\begin{aligned}
& F\left(\xi_{0}, u_{m}, D \phi-D \psi / m, D^{2} \phi\right)+f^{1 / n}(D \phi-D \psi / m)\left(k^{1 / n}\left(\xi_{0}, u_{m}-\psi / m\right)-k^{1 / n}\left(\xi_{0}, u_{m}\right)\right) \\
&=-\left(\operatorname{det} A\left(D \phi-D \psi / m, D^{2} \phi\right)\right)^{1 / n}+f^{1 / n}(D \phi-D \psi / m) k^{1 / n}\left(\xi_{0}, u\right) \\
& \leqslant-\left(\operatorname{det} A\left(D \phi-D \psi / m, D^{2} \phi-D^{2} \psi / m\right)\right)^{1 / n}-\left(\operatorname{det} A\left(D \phi-D \psi / m, D^{2} \psi / m\right)\right)^{1 / n} \\
&+f^{1 / n}(D \phi-D \psi / m) k^{1 / n}\left(\xi_{0}, u\right) \\
&= F\left(\xi_{0}, u, D \phi-D \psi / m, D^{2} \phi-D^{2} \psi / m\right)-\left(\operatorname{det} A\left(D \phi-D \psi / m, D^{2} \psi / m\right)\right)^{1 / n} \\
& \leqslant-\left(\operatorname{det} A\left(D \phi-D \psi / m, D^{2} \psi / m\right)\right)^{1 / n} \leqslant-v / m .
\end{aligned}
$$

Now we shall prove a comparison principle, by assuming that (H6) holds, i.e. $k: \mathbb{R} \rightarrow[0,+\infty)$ is a continuous function which does not depend on $\xi$.

Theorem 4.2. Assume (H2)-(H3) and (H5)-(H6). Let $u \in \operatorname{USC}(\bar{\Omega})$ and $v \in \operatorname{LSC}(\bar{\Omega})$ be respectively a bounded viscosity sub- and supersolution of (DP). Then $u \leqslant v$ in $\bar{\Omega}$.

Proof. We consider $u_{m}=u+\frac{1}{m} \psi$ with $\psi$ as in Lemma 4.1. We may suppose without restriction that $\left(|x|^{2}+\right.$ $\left.|y|^{2}\right) \neq 0$ in $\bar{\Omega}$, otherwise in the definition of $\psi$ we replace $\left(|x|^{2}+|y|^{2}\right)$ with $\left(\left|x-x_{0}\right|^{2}+\left|y-y_{0}\right|^{2}\right)$ with a suitable $\left(x_{0}, y_{0}\right)$. Moreover we choose $g$ in such a way that $\|\psi\|_{\infty}<+\infty$. Our aim is to show that $\sup _{\Omega}\left(u_{m}-v\right) \leqslant$ $\frac{1}{m}\|\psi\|_{\infty}$. Suppose by contradiction that for all $m$ large enough we have $M_{m}=\max _{\bar{\Omega}}\left(u_{m}-v\right)>\frac{1}{m}\|\psi\|_{\infty}$. Since by (H3) we have $u(x) \leqslant \varphi(x) \leqslant v(x)$ for all $x \in \partial \Omega$, such a maximum is achieved at an interior point $\xi_{0}$ (depending on $m$ ). For all $\varepsilon>0$ let us consider the auxiliary function

$$
\Phi_{\varepsilon}(\xi, \zeta)=u_{m}(\xi)-v(\zeta)-\frac{|\xi-\zeta|^{2}}{\varepsilon^{2}}
$$

Let $\left(\xi_{\varepsilon}, \zeta_{\varepsilon}\right)$ be a maximum of $\Phi_{\varepsilon}$ in $\bar{\Omega} \times \bar{\Omega}$. By standard arguments we get, up to subsequences, $\xi_{\varepsilon}, \zeta_{\varepsilon} \rightarrow \tilde{\xi} \in \bar{\Omega}$, and

$$
\begin{aligned}
& \frac{\left|\xi_{\varepsilon}-\zeta_{\varepsilon}\right|^{2}}{\varepsilon^{2}}=o_{\varepsilon}(1) \quad \text { as } \varepsilon \rightarrow 0, \\
& u_{m}\left(\xi_{\varepsilon}\right)-v\left(\zeta_{\varepsilon}\right) \rightarrow u_{m}(\tilde{\xi})-v(\tilde{\xi})=M_{m}, \\
& u_{m}\left(\xi_{\varepsilon}\right) \rightarrow u_{m}(\tilde{\xi}), v\left(\zeta_{\varepsilon}\right) \rightarrow v(\tilde{\xi}) .
\end{aligned}
$$


Since $\tilde{\xi}$ is necessarily in $\Omega$, for $\varepsilon$ small enough we have $\xi_{\varepsilon}, \zeta_{\varepsilon} \in \Omega$ as well. Hence the equation holds for both $u_{m}$ and $v$ respectively in $\xi_{\varepsilon}$ and $\zeta_{\varepsilon}$.

There exist $X, Y \in \mathcal{S}^{N}$ such that, if $p_{\varepsilon}:=2\left(\xi_{\varepsilon}-\zeta_{\varepsilon}\right) / \varepsilon^{2}$, we have

$$
\begin{aligned}
& \left(p_{\varepsilon}, X\right) \in \mathcal{J}^{2,+} u_{m}\left(\xi_{\varepsilon}\right), \quad\left(p_{\varepsilon}, Y\right) \in \mathcal{J}^{2,-} v\left(\zeta_{\varepsilon}\right), \\
& -\frac{8}{\varepsilon^{2}} \mathrm{Id} \leqslant\left(\begin{array}{cc}
X & 0 \\
0 & -Y
\end{array}\right) \leqslant \frac{3}{\varepsilon^{2}}\left(\begin{array}{cc}
I & -I \\
-I & I
\end{array}\right)
\end{aligned}
$$

and by Lemma 4.2

$$
\begin{aligned}
& F\left(\xi_{\varepsilon}, u_{m}, p_{\varepsilon}-D \psi / m, X\right)+f^{1 / n}\left(p_{\varepsilon}-D \psi / m\right)\left(k^{1 / n}\left(u_{m}-\psi / m\right)-k^{1 / n}\left(u_{m}\right)\right)<-\frac{v}{m}, \\
& F\left(\zeta_{\varepsilon}, v, p_{\varepsilon}, Y\right) \geqslant 0 .
\end{aligned}
$$

Moreover, $\left(p_{\varepsilon}-\frac{D \psi}{m}, X-D^{2} \psi / m\right) \in \mathcal{J}^{2,+} u\left(\xi_{\varepsilon}\right)$, and

$$
F_{*}\left(\xi_{\varepsilon}, u\left(\xi_{\varepsilon}\right), p_{\varepsilon}-D \psi / m, X-D^{2} \psi / m\right) \leqslant 0 .
$$

Set $\Sigma_{1}=\sigma\left(p_{\varepsilon}-\frac{1}{m} D \psi\right)$ and $\Sigma_{2}=\sigma\left(p_{\varepsilon}\right)$, where $\sigma$ is the $n \times N$ matrix defined in (4.3). Multiply both sides of the inequality (4.4) by the matrix ( $\left(\begin{array}{ll}\Sigma_{1} & \Sigma_{2}\end{array}\right)$ on the left, and by the transpose of its conjugate on the right, to get

$$
\Sigma_{1} X \bar{\Sigma}_{1}^{\mathrm{T}}-\Sigma_{2} Y \bar{\Sigma}_{2}^{\mathrm{T}} \leqslant \frac{3}{\varepsilon^{2}}\left(\Sigma_{1}-\Sigma_{2}\right)\left(\overline{\Sigma_{1}-\Sigma_{2}}\right)^{\mathrm{T}}=\frac{1}{\varepsilon^{2}} \eta \otimes \bar{\eta}
$$

with

$$
\begin{aligned}
\eta & =\left[a\left(p_{\varepsilon}-\frac{1}{m} D \psi\right)-a\left(p_{\varepsilon}\right)\right]-\mathrm{i}\left[b\left(p_{\varepsilon}-\frac{1}{m} D \psi\right)-b\left(p_{\varepsilon}\right)\right] \\
& =-\frac{g^{\prime}}{m}\left(\frac{y-x\left(p_{\varepsilon}\right)_{2 n+1}-\mathrm{i}\left(x+y\left(p_{\varepsilon}\right)_{2 n+1}\right)}{1+\left(p_{\varepsilon}\right)_{2 n+1}^{2}}\right)=\frac{g^{\prime}}{m}\left(\frac{\left(p_{\varepsilon}\right)_{2 n+1}-\mathrm{i}}{1+\left(p_{\varepsilon}\right)_{2 n+1}^{2}}\right)(x-\mathrm{i} y) .
\end{aligned}
$$

Thus,

$$
\Sigma_{1} X \bar{\Sigma}_{1}^{\mathrm{T}}-\Sigma_{2} Y \bar{\Sigma}_{2}^{\mathrm{T}} \leqslant \frac{\left(g^{\prime}\right)^{2}}{\varepsilon^{2} m^{2}\left(1+\left(p_{\varepsilon}\right)_{2 n+1}^{2}\right)}\left(\|x\|^{2}+\|y\|^{2}\right) \text { Id } .
$$

From (4.6) it follows that

$$
\Sigma_{1}\left(X-D^{2} \psi / m\right) \bar{\Sigma}_{1}^{\mathrm{T}} \geqslant 0
$$

and

$$
\Sigma_{1} X \bar{\Sigma}_{1}^{\mathrm{T}} \geqslant \frac{\Sigma_{1} D^{2} \psi \bar{\Sigma}_{1}^{\mathrm{T}}}{m}=\frac{1}{m}\left(g^{\prime \prime}(x-\mathrm{i} y) \otimes(x+\mathrm{i} y)+2 g^{\prime} \mathrm{Id}\right)>0 .
$$

We will choose the function $g$ in a such way that, for $m$ large enough and for all $\varepsilon$, we have

$$
\left(\frac{g^{\prime \prime}}{m}-\frac{\left(g^{\prime}\right)^{2}}{m^{2} \varepsilon^{2}\left(1+\left(p_{\varepsilon}\right)_{2 n+1}^{2}\right)}\right) \geqslant 0 \text {. }
$$

Thus the following estimate holds

$$
\begin{aligned}
\Sigma_{2} Y \bar{\Sigma}_{2}^{\mathrm{T}} & \geqslant \Sigma_{1} X \bar{\Sigma}_{1}^{\mathrm{T}}-\frac{1}{\varepsilon^{2}} \eta \otimes \bar{\eta} \geqslant \frac{1}{m}\left(g^{\prime \prime}(x-\mathrm{i} y) \otimes(x+\mathrm{i} y)+2 g^{\prime} \mathrm{Id}\right)-\frac{1}{\varepsilon^{2}} \eta \otimes \bar{\eta} \\
& =\left(\frac{g^{\prime \prime}}{m}-\frac{\left(g^{\prime}\right)^{2}}{m^{2} \varepsilon^{2}\left(1+\left(p_{\varepsilon}\right)_{2 n+1}^{2}\right)}\right)(x-\mathrm{i} y) \otimes(x+\mathrm{i} y)+\frac{2 g^{\prime}}{m} \mathrm{Id} \geqslant \frac{2 g^{\prime}}{m} \mathrm{Id}=\gamma \mathrm{Id}>0 .
\end{aligned}
$$


Now, set

$$
\lambda=\frac{\left(g^{\prime}\right)^{2}}{\varepsilon^{2} m^{2}\left(1+\left(p_{\varepsilon}\right)_{2 n+1}^{2}\right)}\left(\|x\|^{2}+\|y\|^{2}\right),
$$

we have

$$
\begin{aligned}
& \left(\operatorname{det}\left(\Sigma_{1} X \Sigma_{1}^{\mathrm{T}}\right)\right)^{1 / n}-\left(\operatorname{det}\left(\Sigma_{2} Y \Sigma_{2}^{\mathrm{T}}\right)\right)^{1 / n} \\
& \quad \leqslant\left(\operatorname{det}\left(\Sigma_{2} Y \Sigma_{2}^{\mathrm{T}}+\lambda \mathrm{Id}\right)\right)^{1 / n}-\left(\operatorname{det}\left(\Sigma_{2} Y \Sigma_{2}^{\mathrm{T}}\right)\right)^{1 / n}=\left(\operatorname{det}\left(\Sigma_{2} Y \Sigma_{2}^{\mathrm{T}}\right)\right)^{1 / n}\left(\operatorname{det}\left(\operatorname{Id}+\lambda\left(\Sigma_{2} Y \Sigma_{2}^{\mathrm{T}}\right)^{-1}\right)^{1 / n}-1\right) \\
& \quad \leqslant\left(\operatorname{det}\left(\Sigma_{2} Y \Sigma_{2}^{\mathrm{T}}\right)\right)^{1 / n}\left(\frac{\operatorname{trace}\left(\operatorname{Id}+\lambda\left(\Sigma_{2} Y \Sigma_{2}^{\mathrm{T}}\right)^{-1}\right)}{n}-1\right) \leqslant \frac{8}{\varepsilon^{2}}\left(\operatorname{det}\left(\Sigma_{2} \Sigma_{2}^{\mathrm{T}}\right)\right)^{1 / n}\left(\frac{\operatorname{trace}\left(\operatorname{Id}+\lambda(\gamma)^{-1}\right)}{n}-1\right) \\
& \quad \leqslant \frac{8}{\varepsilon^{2}}\left(\operatorname{det}\left(\Sigma_{2} \Sigma_{2}^{\mathrm{T}}\right)\right)^{1 / n}\left(\frac{\lambda m}{2 g^{\prime}}\right) \leqslant \frac{16}{\varepsilon^{2}}\left(1+\frac{1}{2}\left(\left|a\left(p_{\varepsilon}\right)\right|^{2}+\left|b\left(p_{\varepsilon}\right)\right|^{2}\right)\right)^{1 / n}\left(\frac{g^{\prime}\left(\|x\|^{2}+\|y\|^{2}\right)}{2 m \varepsilon^{2}\left(1+\left(p_{\varepsilon}\right)_{2 n+1}^{2}\right)}\right) \\
& \quad \leqslant \frac{C}{\varepsilon^{2} \varepsilon^{2 / n}}\left(\frac{g^{\prime}\left(\|x\|^{2}+\|y\|^{2}\right)}{2 m \varepsilon^{2}}\right),
\end{aligned}
$$

where, in the last inequality, we use the fact that $\left|p_{\varepsilon}\right| \leqslant C / \varepsilon$, for some $C>0$ independent of $\varepsilon, m$. Set $\xi=(x, y, 0)$, we also have

$$
\begin{aligned}
f^{1 / n}\left(p_{\varepsilon}\right)-f^{1 / n}\left(p_{\varepsilon}-\frac{D \psi}{m}\right) & =f^{1 / n}\left(p_{\varepsilon}\right)-f^{1 / n}\left(p_{\varepsilon}-g^{\prime} \xi / m\right) \\
& \leqslant C \frac{1}{\varepsilon^{2 / n}} \frac{g^{\prime}}{m}\left(\|x\|^{2}+\|y\|^{2}\right)^{1 / 2}\left(1+o_{\varepsilon}(1)\right) .
\end{aligned}
$$

By subtracting the two inequalities in (4.5) and by using (H2), (H5), (4.9) and (4.10), we finally obtain

$$
\begin{aligned}
\frac{v}{m} & =\frac{2 g^{\prime}\left(1+\left(g^{\prime \prime} / 2 g^{\prime}\right)\left(\|x\|^{2}+\|y\|^{2}\right)\right)^{1 / n}}{m} \\
& \leqslant \frac{C}{\varepsilon^{2 / n}} \frac{g^{\prime}}{m}\left(\|x\|^{2}+\|y\|^{2}\right)^{1 / 2}\left(1+o_{\varepsilon}(1)\right)+\frac{C}{\varepsilon^{2} \varepsilon^{2 / n}}\left(\frac{g^{\prime}\left(\|x\|^{2}+\|y\|^{2}\right)}{2 m \varepsilon^{2}}\right) .
\end{aligned}
$$

Now we take $g(s)=\exp (\beta s-\alpha)$ with $\beta$ and $\alpha$ to be determined as follows. We have $g^{\prime}=\beta g$, and $g^{\prime \prime}=\beta^{2} g$. Since $(x, y) \neq(0,0)$, if we choose $m=\beta^{(2 n+1) /(2 n)}$ and $\varepsilon=\beta^{-1 /(6 n)}$, then for $\beta$ large enough we get a contradiction in (4.11). We finally take $\alpha$ (depending on $\beta$ and the diameter of $\Omega$ ) in such a way that $g \leqslant 1$. We point out that by this choice of $g, \varepsilon$ and $m$ the inequality (4.8) is satisfied. Thus we can conclude.

Remark 4.1. One can prove a variant of Theorems 4.1 and 4.2 in which the condition $u \leqslant v$ on $\partial \Omega$ is dropped and the conclusion is changed to $u-v \leqslant \sup _{\partial \Omega}(u-v)^{+}$(see e.g. User's guide [9]).

In the general case when $k$ depends on $x$ and it is not strict monotone with respect to $u$ we are able to prove a comparison result between continuous sub and supersolution of (DP) by following a dilation argument (see e.g [24, Theorem 2.2], [16,4]).

Theorem 4.3. Assume (H5) and (H7) and suppose that $k>0$. Let $u, v \in C(\bar{\Omega})$ be respectively viscosity sub-and supersolution of (DP). Then

$$
\sup _{\Omega}(u-v) \leqslant \sup _{\partial \Omega}(u-v)^{+} .
$$


Proof. Let $R=\max \left(\|u\|_{\infty},\|v\|_{\infty}\right)$. It is not restrictive to assume that $u$ is nonnegative in $\Omega$ and $|\xi|<\operatorname{diam}(\Omega)$ for every $\xi \in \Omega$. Actually we may always replace $F$ by $G(\xi, s, p, X)=F\left(\xi-\xi_{0}, s-R, p, X\right)$. For a suitable $\xi_{0} \in \mathbb{R}^{N}$.

For all $r>1$ we set $\Omega_{r}=r^{-1} \Omega$ and we introduce the function

$$
u_{r}(x)=r^{-1} u(r x), \quad x \in \bar{\Omega}_{r} .
$$

We claim that there is $\delta<0$ such that for all $r>1$ close to $1, u_{r}$ is a viscosity solution of

$$
F\left(x, u_{r}(x), D u_{r}, D^{2} u_{r}\right) \leqslant-\delta(r-1) \quad \text { in } \Omega_{r} .
$$

Indeed let $\phi \in C^{2}\left(\bar{\Omega}_{r}\right)$ and $\xi \in \Omega_{r}$ such that $u_{r}-\phi$ has a local maximum at $\xi$. Then $u(y)-r \phi\left(r^{-1} y\right)$ has a local maximum at $r \xi$. Since $u$ is a subsolution of (DP) we have

$$
F_{*}\left(r \xi, u(r \xi), D \phi, r^{-1} D^{2} \phi\right) \leqslant 0 .
$$

The above inequality implies that $L \phi(\xi) \geqslant 0$ and

$$
-\operatorname{det} A\left(D \phi, D^{2} \phi\right)+r^{n} k(r \xi, u(r \xi)) f(D \phi) \leqslant 0 .
$$

Set $L_{\Omega}=\sup _{\Omega \times \Omega \times[0, R]}\left(\left(k(\xi, u)-k\left(\xi_{0}, u\right)\right) /\left|\xi-\xi_{0}\right|\right)$ and suppose for the moment that

$$
L_{\Omega}<\frac{n \inf _{\Omega \times \mathbb{R}} k}{\operatorname{diam}(\Omega)} .
$$

The following estimate holds

$$
\begin{aligned}
- & \operatorname{det} A\left(D \phi, D^{2} \phi\right)+k\left(\xi, u_{r}(\xi)\right) f(D \phi) \\
\leqslant & k\left(\xi, u_{r}(\xi)\right) f(D \phi)-r^{n} k(r \xi, u(r \xi)) f(D \phi) \\
= & \left(1-r^{n}\right) k(r \xi, u(r \xi)) f(D \phi)+\left[k\left(\xi, u_{r}(\xi)\right)-k\left(\xi, r u_{r}(\xi)\right)\right] f(D \phi) \\
& +\left[k\left(\xi, r u_{r}(\xi)\right)-k(r \xi, u(r \xi))\right] f(D \phi) \\
\leqslant & f(D \phi)\left[\left(1-r^{n}\right)\left(\inf _{\bar{\Omega} \times \mathbb{R}} k\right)+(r-1) L_{\Omega}|\xi|\right] \\
\leqslant & (r-1) f(D \phi)\left[-n\left(\inf _{\bar{\Omega} \times \mathbb{R}} k\right)+L_{\Omega} \operatorname{diam}(\Omega)\right] .
\end{aligned}
$$

From (4.13) and the fact that $f(D \phi) \geqslant 1$, it follows that there is $\delta>0$ such that we have

$$
-\operatorname{det} A\left(D \phi, D^{2} \phi\right)+k\left(\xi, u_{r}(\xi)\right) f(D \phi) \leqslant-\delta(r-1) .
$$

Now by arguing as in the proof of Theorem 4.1 one gets

$$
\sup _{\Omega \cap \Omega_{r}}\left(u_{r}-v\right) \leqslant \sup _{\partial\left(\Omega \cap \Omega_{r}\right)}\left(u_{r}-v\right)^{+}
$$

and the conclusion follows by letting $r \rightarrow 1^{+}$.

If (4.13) is not satisfied one proceeds by covering $\Omega$ with small balls of radius $r<L_{\Omega}{ }^{-1} \inf _{\Omega \times \mathbb{R}} k$.

Remark 4.2. We remark that Theorem 4.3 is a consequence of the fact that we are considering a curvature equation. Precisely set $\rho_{r}(z)=\rho(r z)$, with $\rho(z)=u(\xi)-s$ and let $K$ the Levi curvature of $\{\rho=0\}$. Then we have

$$
\partial_{z} \rho_{r}(z)=r\left(\partial_{z} \rho\right)(r z), \quad \partial_{z \bar{z}} \rho_{r}(z)=r^{2}\left(\partial_{z \bar{z}} \rho\right)(r z)
$$

and

$$
-\left\{\left|\partial \rho_{r}\right|^{-n-2} \operatorname{det}\left(\begin{array}{cc}
0 & \partial_{\bar{z}} \rho_{r} \\
\partial_{z} \rho_{r} & \partial_{z \bar{z}} \rho_{r}
\end{array}\right)\right\}(z)=-r^{n}\left\{|\partial \rho|^{-n-2} \operatorname{det}\left(\begin{array}{cc}
0 & \partial_{\bar{z}} \rho \\
\partial_{z} \rho & \partial_{z \bar{z}} \rho
\end{array}\right)\right\}(r z)=r^{n} K(r z) \text {. }
$$


By the comparison results and the Perron's method we get the existence of a unique continuous solution of (DP).

Corollary 4.1. Assume either the hypotheses of Theorem 4.1 or of Theorem 4.2 and suppose (H4) holds. Then for any $\varphi \in C(\partial \Omega)$ there exists a unique continuous viscosity solution of (DP).

Proof. We use the Perron's method introduced for viscosity solutions by H. Ishii [15] with the version up to the boundary of the first author [10]. We observe that if $M$ is large enough then the function $\bar{v}(\xi)=M$ is a supersolution of (DP). Let us denote by $\xi_{0}$ the center of the sphere of minimum radius $r$ containing $\bar{\Omega}$. Under the assumption (H4) the function $\underline{v}(\xi)=\left(r^{2}-\left|\xi-\xi_{0}\right|^{2}\right)^{1 / 2}-M$ is subsolution of (DP). Indeed if we set $\rho(\xi, s)=$ $\left(r^{2}-\left|\xi-\xi_{0}\right|^{2}\right)^{1 / 2}-M-s$ then the zero level set of $\rho$ is a subset of a sphere of radius $r$ and one knows that in this case $k_{\{\rho=0\}}(z)=1 / r^{n}$. Thus $F\left(\xi, \underline{v}(\xi), D \underline{v}(\xi), D^{2} \underline{v}(\xi)\right)=-1 / r+k^{1 / n}(\xi, \underline{v}(\xi))<0$.

The Perron's method provides us with a (possibly discontinuous) solution $u$ of (DP) such that $\underline{v} \leqslant u \leqslant \bar{v}$ in $\bar{\Omega}$. The condition that $\Sigma=\emptyset$ implies that there is no loss of boundary condition on $\partial \Omega$ and therefore, every subsolution $\omega$ and every supersolution $w$ of (DP) satisfies

$$
\omega \leqslant \varphi \leqslant w \text { on } \partial \Omega \text {. }
$$

The first consequence of this inequality is that $u_{*}=u^{*}=\varphi$ on $\partial \Omega$ and therefore $u$ is continuous at points of $\partial \Omega$. The second one is the uniqueness of the continuous solution $u$ of (DP) which follows from either Theorem 4.1 or Theorem 4.2.

\section{Lipschitz estimates and proofs of Theorems 1.1, 1.2, 1.3}

In this section we denote by $x$ a point in $\mathbb{R}^{N}$, with $N=2 n+1$. We show the existence of a Lipschitz continuous viscosity solution of (DP) under suitable assumptions on $k$ and geometric conditions on the domain. To this purpose we follow two different approaches. More precisely in the particular case that $k$ does not depend on the variable $x$ we adapt the method of translation (see e.g. [16]), whereas in the case when $k$ depends on $x$ and $u$, since it is not possible in general to obtain the existence through the Perron's method we use a Bernstein type method and a proper approximation argument that we explain later.

Throughout this section we assume that $\Omega$ satisfies (H3). We recall that under (H3) the boundary data are assumed in a classical sense by the viscosity subsolutions and supersolutions of (DP), and the conditions (3.12) and (3.13) are satisfied.

We introduce the following notation: for $\gamma>0$ we set

$$
\Omega_{\gamma}:=\{x \in \bar{\Omega}: d(x)<\gamma\} .
$$

We observe that since $\partial \Omega$ is of class $C^{2}$ then for $\gamma>0$ small the distance function $d \in C^{2}\left(\Omega_{\gamma}\right)$.

We start with the following lemma.

Lemma 5.1. Assume (H3), $\varphi \in C^{1,1}(\partial \Omega)$. Then there are $\lambda^{\prime}>0$, and $0<\gamma^{\prime} \leqslant \gamma$ such that for all $\lambda \geqslant \lambda^{\prime}$ the functions $\bar{u}(x)=\varphi(x)-\lambda d(x)$, and $\bar{u}(x)=\varphi(x)+\lambda d(x)$ are respectively classical subsolution and supersolution of (DP) in $\Omega_{\gamma^{\prime}}$ and $\bar{u}(x)=\bar{u}(x)=\varphi(x)$ in $\partial \Omega$. Moreover $\bar{u}$ and $\bar{u}$ are Lipschitz continuous in $\bar{\Omega}_{\gamma}$.

Proof. Let us continue to denote by $\varphi$ the smooth extension of $\varphi$ to $\bar{\Omega}$.

Subsolution case: We show that there are $\gamma^{\prime} \leqslant \gamma$ and $\lambda^{\prime}>0$ such that for all $\lambda \geqslant \lambda^{\prime}, \bar{u}(x)$ is a classical subsolution of (DP) in $\Omega_{\gamma^{\prime}}$. We have $D \bar{u}(x)=D \varphi(x)-\lambda D d(x), D^{2} \bar{u}(x)=D^{2} \varphi(x)-\lambda D^{2} d(x)$. From the condition (3.12) and the continuity of $A_{\infty}$ there is $r_{0}>0$ such that for all $x \in B\left(x_{0}, r_{0}\right)$ we have $A_{\infty}\left(-D d(x),-D^{2} d(x)\right)>0$. We notice that

$$
A\left(D \bar{u}(x), D^{2} \bar{u}(x)\right)=\frac{\lambda}{\left.\left[\left(\lambda^{-2}\right)+\left(\lambda^{-1} D \bar{u}(x)\right)\right)_{N}^{2}\right]^{2}} A^{\prime}\left(\lambda^{-1} D \bar{u}(x), \lambda^{-1} D^{2} \bar{u}(x), \lambda\right)
$$


and $A^{\prime}\left(\lambda^{-1} D \bar{u}(x), \lambda^{-1} D^{2} \bar{u}(x), \lambda\right)$ converges to $A_{\infty}\left(D d(x), D^{2} d(x)\right)$ uniformly in $B\left(x_{0}, r_{0}\right)$ as $\lambda \rightarrow \infty$. Thus there exists $\lambda_{0}:=\lambda\left(x_{0}, r\right)$ such that for all $\lambda \geqslant \lambda_{0}$ and for all $x \in B\left(x_{0}, r_{0}\right)$ the matrix $A^{\prime}\left(\lambda^{-1} D \varphi(x)-\right.$ $\left.D d(x), \lambda^{-1} D^{2} \varphi(x)-D^{2} d(x)\right)$ is definite positive as well. Since $\partial \Omega$ is compact, we can find $\gamma^{\prime}<\gamma$ and $\lambda^{\prime}$ such that for all $\lambda \geqslant \lambda^{\prime}$ and for all $x \in \Omega_{\gamma^{\prime}}$ the matrix the matrix $A\left(D \bar{u}(x), D^{2} \bar{u}(x)\right)$ is definite positive.

On the other hand one sees that

$$
h(D \bar{u}(x)) \operatorname{det}\left(B\left(D \bar{u}(x), D^{2} \bar{u}(x)\right)\right) \rightarrow 2^{n} \operatorname{det}\left(B_{\infty}\left(D d(x), D^{2} d(x)\right)\right)
$$

as $\lambda \rightarrow \infty$ uniformly in $x \in B\left(x_{0}, r_{0}\right)$. Since $\partial \Omega$ is a compact set, the condition (3.13) implies that for $\lambda$ large enough and for all $x \in \Omega_{\gamma^{\prime}}$ we have

$$
\left(-h(D \bar{u}(x)) \operatorname{det} B\left(D \bar{u}(x), D^{2} \bar{u}(x)\right)\right)^{1 / n}>k^{1 / n}(x, \bar{u}(x))
$$

and in particular

$$
\left(\operatorname{det} A\left(D \bar{u}(x), D^{2} \bar{u}(x)\right) f^{-1}(D \bar{u}(x))\right)^{1 / n}>k^{1 / n}(x, \bar{u}(x)) .
$$

Thus for $\lambda>0$ large enough and for all $x \in \Omega_{\gamma^{\prime}}$ we get

$$
\begin{aligned}
& F_{*}\left(x, \bar{u}(x), D \bar{u}(x), D^{2} \bar{u}(x)\right) \\
& \quad=f^{1 / n}(D \bar{u}(x)) \cdot\left\{\left(-\operatorname{det} A\left(D \bar{u}(x), D^{2} \bar{u}(x)\right) f^{-1}(D \bar{u}(x))\right)^{1 / n}+k^{1 / n}(x, \bar{u})\right\}<0 .
\end{aligned}
$$

This proves that $\bar{u}$ is a classical subsolution of (DP) in $\Omega_{\gamma^{\prime}}$.

Supersolution case: Let us consider the function $\bar{u}(x)=\varphi(x)+\lambda d(x)$. We first notice that for every $x_{0} \in \partial \Omega, A_{\infty}\left(D d, D^{2} d\right)=-A_{\infty}\left(-D d,-D^{2} d\right)$ is not semidefinite positive. This means that there exists at least one eigenvalue which is strictly negative. By analogous arguments as above one can show that for $\lambda>0$ large enough and for $\gamma^{\prime}$ small the matrix $A\left(D \varphi(x)+\lambda D d(x), D^{2} \varphi(x)+\lambda D^{2} d(x)\right)$ is not semidefinite positive for all $x \in \Omega_{\gamma^{\prime}}$. This implies that $F^{*}\left(x, \bar{u}, D \bar{u}, D^{2} \bar{u}\right)=+\infty$. Finally the Lipschitz continuity of $\bar{u}$ and $\bar{u}$ follows from the fact that $\varphi \in C^{1,1}\left(\bar{\Omega}_{\gamma}\right)$ and $d \in C^{2}\left(\bar{\Omega}_{\gamma}\right)$. Thus we can conclude.

Next we prove the Lipschitz continuity of the solution to (DP) under the assumption that $k$ does not depend on $x$.

Theorem 5.1 (The $x$-independent case). Assume (H2)-(H6), $\varphi \in C^{1,1}(\partial \Omega)$. Then there exists a unique Lipschitz continuous viscosity solution $u$ of (DP).

Proof. Let us continue to denote by $\varphi$ the smooth extension of $\varphi$ to $\bar{\Omega}$. The existence of a continuous solution $u$ to (DP) follows from Corollary 4.1. Moreover by comparing $u$ with the barriers defined in Corollary 4.1 one gets that $\|u\|_{\infty}<R$ for some $R>0$. Now we consider the functions $\bar{u}$ and $\bar{u}$ defined in Lemma 5.1. We have $\bar{u}=u=\bar{u}$ on $\partial \Omega$, and $\bar{u} \leqslant u \leqslant \bar{u}$ on $d(x)=\gamma^{\prime}$ provided $\lambda>\left(\|u\|_{\infty}+\|\varphi\|_{\infty}\right) / \gamma^{\prime}$. Theorem 4.2 yields that $\bar{u} \leqslant u \leqslant \bar{u}$ in $\Omega_{\gamma^{\prime}}$. To show the Lipschitz continuity of $u$ we adapt the method of translations (see [16]). Given $h \in \mathbb{R}^{N}$, the function $u(\cdot+h)$ is a viscosity solution of the same equation as that for $u$ but set in $\Omega-h$, since the equation does not depend on $x$. Theorem 4.2 and Remark 4.1 yield

$$
\begin{aligned}
\sup _{\Omega_{\gamma^{\prime}} \cap\left(\Omega_{\gamma^{\prime}}-h\right)}|u-u(\cdot+h)| & \leqslant \sup _{\partial\left(\Omega_{\gamma^{\prime}} \cap\left(\Omega_{\gamma^{\prime}}-h\right)\right)}|u-u(\cdot+h)| c \\
& \leqslant \sup _{\partial\left(\Omega_{\gamma^{\prime}} \cap\left(\Omega_{\gamma^{\prime}}-h\right)\right)} \max \{|\bar{u}-\bar{u}(\cdot+h)|,|\bar{u}-\bar{u}(\cdot+h)|\} \\
& \leqslant C|h| .
\end{aligned}
$$


Thus $u$ is Lipschitz continuous in $\Omega_{\gamma^{\prime}}$. Next we show that this implies that $u$ is Lipschitz continuous in $\Omega$. Indeed by Theorem 4.2 and Remark 4.1 we have

$$
\sup _{\Omega \cap(\Omega-h)}|u-u(\cdot+h)| \leqslant \sup _{\partial(\Omega \cap(\Omega-h))}|u-u(\cdot+h)| .
$$

We estimate the 1.h.s of (5.1). If $h \leqslant \gamma^{\prime}$, then $\sup _{\partial(\Omega \cap(\Omega-h))}|u-u(\cdot+h)| \leqslant C|h|$ by the above estimates. If $h>\gamma^{\prime}$ we have $|u(x)-u(x+h)| \leqslant 2\|u\|_{\infty} \leqslant 2\|u\|_{\infty} \frac{h}{\gamma^{\prime}}$. In any case we get

$$
\frac{\sup }{\Omega \cap(\Omega-h)}|u-u(\cdot+h)| \leqslant C|h|,
$$

and we can conclude.

Proof of Theorem 1.2. In view of (2.3) and of (2.4), the Dirichlet problem (1.2) is equivalent to (DP). Hence Theorem 1.2 follows from Theorem 5.1.

Next we prove the existence of a Lipschitz continuous solution to (DP) under more general conditions on $k$ for which we cannot applying directly the Perron's method not having a comparison result. To this end we use a "weak Bernstein method" introduced by Barles in [3] to obtain gradient bound for viscosity solutions to fully nonlinear pde's. Roughly speaking in [3] it is shown that if a continuous degenerate elliptic operator $G: \bar{\Omega} \times \mathbb{R} \times \mathbb{R} \times \mathcal{S}^{N} \rightarrow \mathbb{R}$ satisfies in a neighborhood of the set of $\{(x, u, p, M):|u| \leqslant R,|p| \geqslant L, G=0\}$ the condition

$$
D_{x} G \cdot p+D_{u} G|p|^{2}-g D_{M} G \cdot M^{2}>\alpha>0
$$

for some constants $\alpha, g>0$ and $L$ large and for all $R>0$ then any viscosity solution of $G=0$ satisfies

$$
\sup _{\bar{\Omega}}|D u(x)| \leqslant \max \left(L, \sup _{\partial \Omega}|D u(x)|\right) .
$$

Next we will use the hypothesis (H8).

Remark 5.1. We observe that

(i) if $k \in C^{1}(\Omega \times \mathbb{R},[0,+\infty))$, then (H8) is satisfied if for instance one the following conditions hold:

(1) $D_{u}>0$

(2) $\left|D_{x} k\right| \leqslant L D_{u} k+\tilde{g} n k^{1+1 / n}$ for some $L>0$ and $\tilde{g}<g_{0}$.

(ii) if $k$ satisfies (H5), (H6) and (H7), then it satisfies (H8) too. Hence, the following approach provides an alternative proof of Theorem 5.1 for Lipschitz continuous $k$.

We first show that if $k \in C^{1}(\Omega \times \mathbb{R},[0,+\infty))$ satisfies (H8) for some $\alpha>0$, then a Lipschitz continuous solution of (DP) satisfies (5.3).

To this end we denote

$$
\widetilde{F}(x, u, p, X)=-\operatorname{det}(A(p, X))+k(x, u) f(p) .
$$

We start with the following lemma in which we prove that if $k \in C^{1}(\Omega \times \mathbb{R},[0,+\infty))$ satisfies (H8) for some $\alpha>0$, then the operator $\widetilde{F}$ satisfies the condition (5.2) in a neighborhood of the set $V(R, L)=\{(x, u, p, M)$ : $|u| \leqslant R,|p| \geqslant L, F(x, u, p, M)=0\}$.

Lemma 5.2. If $k \in C^{1}(\Omega \times \mathbb{R},[0,+\infty))$ satisfies (H8) for some $\alpha>0$, then the condition (5.2) is satisfied by $\widetilde{F}$ in a neighborhood $W(R, L)$ of the set $V(R, L)$. 
Proof. We start by showing that (5.2) holds in the set $V(R, L)$.

We first observe that if $(x, u, p, M) \in V(R, L)$ then we have $A(p, X) \geqslant 0$ and $\widetilde{F}(x, u, p, X)=-\operatorname{det} A(p, X)+$ $k(x, u) f(p)=0$. The following equalities hold.

$$
D_{x} \widetilde{F}=f(p) D_{x} k(x, u), \quad D_{u} \widetilde{F}=f(p) D_{u} k(x, u) .
$$

Now set $\Sigma(p)=\bar{\sigma}^{\mathrm{T}}(p) \sigma(p)$, where $\sigma(p)$ is the matrix defined in (4.3). We notice that $\Sigma(p) \geqslant 0$ with minimum eigenvalue identically zero and

$$
\Sigma(p) \leqslant \operatorname{Tr} \Sigma(p) \cdot I_{N}
$$

where $\operatorname{Tr} \Sigma(p)=2 n+|a(p)|^{2}+|b(p)|^{2}=2 n+\left(\sum_{1}^{2 n} p_{j}^{2}\right) /\left(1+p_{N}^{2}\right)$.

By denoting $A^{*}(p, X)$ the cofactor matrix of $A(p, X)$, recalling that $\operatorname{det} A^{*}(p, X)=(\operatorname{det} A(p, X))^{n-1}$ and using the inequality (5.5), we have

$$
\begin{aligned}
-D_{M} \widetilde{F} \cdot M^{2} & =\operatorname{Tr}\left[\left(A^{*}(p, M)\right) \sigma(p) M^{2} \bar{\sigma}^{\mathrm{T}}(p)\right] \geqslant n\left(\operatorname{det}\left[\left(A^{*}(p, M)\right)(p) \sigma(p) M^{2} \bar{\sigma}^{\mathrm{T}}\right]\right)^{1 / n} \\
& \geqslant n[\operatorname{Tr} \Sigma(p)]^{-1 / n}\left(\operatorname{det}\left[\left(A^{*}(p, M)\right) \sigma(p) M \Sigma(p) M \bar{\sigma}^{\mathrm{T}}(p)\right]\right)^{1 / n} \\
& =n \operatorname{det}(A(p, M))^{1+1 / n}[\operatorname{Tr} \Sigma(p)]^{-1 / n}=n(k(x, u) f(p))^{1+1 / n}[\operatorname{Tr} \Sigma(p)]^{-1 / n} .
\end{aligned}
$$

In (5.6) we use the fact that for all Hermitian matrices $A, B \geqslant 0$ we have

$$
\operatorname{det}(A B) \leqslant\left(\frac{\operatorname{Tr}(A B)}{n}\right)^{n} \text {. }
$$

Moreover we have

$$
\begin{aligned}
f^{1 / n}(p)[\operatorname{Tr} \Sigma(p)]^{-1 / n} & =\left[2 \frac{\left(1+|p|^{2}\right)^{(n+2) /(2 n)}}{\left(1+p_{N}^{2}\right)^{1 / n}}\right]\left[\frac{\left(1+p_{N}^{2}\right)^{1 / n}}{\left(2 n+|p|^{2}+(2 n-1) p_{N}^{2}\right)^{1 / n}}\right] \\
& \geqslant 2 \frac{\left(1+|p|^{2}\right)^{(n+2) / 2 n)}}{\left(2^{n}\left(1+|p|^{2}\right)\right)^{1 / n}} \geqslant\left(1+|p|^{2}\right)^{1 / 2} .
\end{aligned}
$$

We set

$$
I(x, u, p)=\frac{D_{x} k \cdot p+D_{u} k|p|^{2}}{\left(1+|p|^{2}\right)^{1 / 2}}+g n k^{1+1 / n} .
$$

We recall $k \in C^{1}(\Omega \times \mathbb{R},[0,+\infty))$ and therefore the map $I$ is a continuous function. By combining the above estimates and using the fact that $f(p)\left(1+|p|^{2}\right)^{1 / 2} \geqslant 1$, for all $(x, u, p, M) \in V(R, L)$ we obtain

$$
\begin{aligned}
& D_{x} \widetilde{F} \cdot p+D_{u} \widetilde{F}|p|^{2}-g_{0} D_{M} \widetilde{F} \cdot M^{2} \\
& \quad=f(p) D_{x} k(x, u) \cdot p+f(p) D_{u} k(x, u)+g_{0} n(k(x, u) f(p))^{1+1 / n}[\operatorname{Tr} \Sigma(p)]^{-1 / n} \\
& \quad \geqslant f(p)\left(1+|p|^{2}\right)^{1 / 2} I(x, u, p) \geqslant \alpha>0 .
\end{aligned}
$$

The above estimate holds in a neighborhood of $V(R, L)$ by continuity.

Proposition 5.1 (Weak Bernstein Method). Assume that $k \in C^{1}(\Omega \times \mathbb{R},[0,+\infty))$ satisfies (H5) and (H8) for some $\alpha>0$. Let $u$ be a continuous solution of (DP) such that

$$
|u(x)-u(w)| \leqslant K|x-w|, \quad \text { for all }(x, w) \in \partial(\Omega \times \Omega) .
$$

Then we have

$$
|u(x)-u(w)| \leqslant \bar{C}|x-w|, \quad \text { for all }(x, w) \in \Omega \times \Omega
$$

where $\bar{C}=\max (L, K)$. 
Proof. We follow the arguments of [3, Theorem 1]. We consider the function

$$
\Phi(x, w):=u(x)-u(w)-C|x-w| .
$$

We assume by contradiction that for all $C>\max (L, K)$ there is a point $(x, w)$ such that $\Phi(x, w)>0$. We observe that because of (5.9), if $C>0$ is large then $(x, w) \in \Omega \times \Omega$. We set $\psi(x-w)=C|x-w|$. There exist $X, Y \in \mathcal{S}(N)$ such that, if $p:=D \psi(x-w)$, we have

$$
\begin{aligned}
& (p, X) \in \mathcal{J}^{2,+} u(x), \quad(p, Y) \in \mathcal{J}^{2,-} u(w), \\
& \left(\begin{array}{cc}
X & 0 \\
0 & -Y
\end{array}\right) \leqslant\left(\begin{array}{cc}
D^{2} \psi(x-w) & -D^{2} \psi(x-w) \\
-D^{2} \psi(x-w) & D^{2} \psi(x-w)
\end{array}\right)
\end{aligned}
$$

and

$$
F_{*}(x, u(x), p, X) \leqslant 0 \quad \text { and } \quad F^{*}(w, u(w), p, Y) \geqslant 0 .
$$

From (5.11) it follows that $A(p, Y) \geqslant A(p, X) \geqslant 0$. Moreover for all $t \in[0,1]$ we have $A(p, t X+(1-t) Y) \geqslant 0$ as well. Moreover from (5.12) it follows that we have

$$
-\operatorname{det}(A(p, X))+k(x, u(x)) f(p) \leqslant 0 \quad \text { and } \quad-\operatorname{det}(A(p, Y))+k(w, u(w)) f(p) \geqslant 0 .
$$

We set $\widetilde{F}(x, u, p, M)=-\operatorname{det}(A(p, M))+k(x, u) f(p)$. We consider the function $g$ defined by

$$
g(t)=\widetilde{F}(t x+(1-t) w, t u(x)+(1-t) u(w), p, t X+(1-t) Y) .
$$

Since $g(t)$ is continuous there are $t_{1} \leqslant t_{2}$ such that $g\left(t_{1}\right) \geqslant 0, g\left(t_{2}\right) \leqslant 0$ and $g(t) \in W(R, L)$ for all $t \in\left[t_{1}, t_{2}\right]$. We assume $\widetilde{F}$ smooth. We set $\gamma=C /|x-w|$. By using (5.10) and (H5) we get

$$
\begin{aligned}
g^{\prime}(t) & =\frac{1}{\gamma}\left\{D_{x} \widetilde{F} \cdot p+D_{u} \widetilde{F}|p|^{2}+\gamma D_{M} \widetilde{F} \cdot(X-Y)+\gamma D_{u} \widetilde{F} \cdot \Phi(x, w)\right\} \\
& \geqslant \frac{1}{\gamma}\left\{D_{x} \widetilde{F} \cdot p+D_{u} \widetilde{F}|p|^{2}+\gamma D_{M} \widetilde{F} \cdot(X-Y)\right\} .
\end{aligned}
$$

By Lemma 2 in [3] we have

$$
\gamma(Y-X) \geqslant g_{0}(t X+(1-t) Y)^{2} .
$$

Hence from Lemma 5.2 it follows that $g^{\prime}(t)>0$ for $t \in\left[t_{1}, t_{2}\right]$, but this is in contradiction with the fact that $g\left(t_{1}\right) \geqslant 0, g\left(t_{2}\right) \leqslant 0$.

Let us mention that when $k$ is only Lipschitz continuous and (H8) holds with some $\alpha>0$ then the above a priori estimate comes from an approximation argument.

More precisely, let $\tilde{k}$ be the nonnegative function defined on $\mathbb{R}^{N} \times \mathbb{R}$ such that $\tilde{k}(x, u)=k(x, u)$ if $x \in \bar{\Omega}$ and $\tilde{k}(x, u)=2\left(\frac{\alpha}{g n}\right)^{n /(n+1)}$ if $x \notin \bar{\Omega}$. We set

$$
\tilde{I}(x, u, p)=\frac{D_{x} \tilde{k} \cdot p+D_{u} \tilde{k}|p|^{2}}{\left(1+|p|^{2}\right)^{1 / 2}}+g n \tilde{k}^{1+1 / n} .
$$

We denote by $k_{\varepsilon}$ and $I_{\varepsilon}$ the convolution with respect of the variables $(x, u)$ of $\tilde{k}$ and $\tilde{I}$ respectively. With a positive mollifier $J_{\varepsilon}$ and by (DP) $)_{\varepsilon}$ the Dirichlet problem (DP) for $k_{\varepsilon}$. The following result holds.

Corollary 5.1. Assume that $k$ satisfies $(\mathrm{H} 7)$ and that $(\mathrm{H} 8)$ holds for some $\alpha>0$ and for a.e. $(x, u) \in \Omega \times \mathbb{R}$. Suppose that for any $\varepsilon>0$ there is a continuous solution $u_{\varepsilon}$ of $(\mathrm{DP})_{\varepsilon}$. If the family $\left(u_{\varepsilon}\right)$ is equibounded in $\bar{\Omega}$ and there is a positive constant $K$ such that for every $\varepsilon>0$

$$
\left|u_{\varepsilon}(x)-u_{\varepsilon}(w)\right| \leqslant K|x-w|, \quad \text { for all }(x, w) \in \partial(\Omega \times \Omega)
$$


then, up to a subsequence, $u_{\varepsilon}$ uniformly converges to a Lipschitz continuous solution $u$ of (DP) on the compact subsets of $\bar{\Omega}$.

Proof. We claim that if $k$ is Lipschitz continuous and satisfies condition (H8) a.e. then, for all $\varepsilon>0$ small, $k_{\varepsilon}=$ $\tilde{k} * J_{\varepsilon}$ satisfies condition (H8) for some $0<\bar{\alpha}<\alpha$. The thesis will then follow by applying Proposition 5.1 to $u_{\varepsilon}$, by remarking that the constant $\widetilde{C}$ is independent of $\varepsilon$, and by using the Ascoli-Arzelà Theorem. To prove the claim, we observe that by (H8) and by the construction of $\tilde{I}$ we have $\tilde{I}(x, u, p) \geqslant \alpha$, for almost every $x \in \mathbb{R}^{N},|u| \leqslant R$, $|p| \geqslant L$. Moreover since $k_{\varepsilon}$ converge uniformly to $k$ for all $x \in \bar{\Omega},|u| \leqslant R$ then for all $x \in \bar{\Omega},|u| \leqslant R,|p| \geqslant L$ we have

$$
\begin{aligned}
\alpha \leqslant I_{\varepsilon}(x, u, p) & =\frac{D_{x} k_{\varepsilon} \cdot p+D_{u} k_{\varepsilon}|p|^{2}}{\left(1+|p|^{2}\right)^{1 / 2}}+g n\left(k^{1+1 / n} * J_{\varepsilon}\right)(x, u) \\
& =\frac{D_{x} k_{\varepsilon} \cdot p+D_{u} k_{\varepsilon}|p|^{2}}{\left(1+|p|^{2}\right)^{1 / 2}}+g n k_{\varepsilon}^{1+1 / n}+g n\left(\left(k^{1+1 / n} * J_{\varepsilon}\right)(x, u)-k_{\varepsilon}^{1+1 / n}(x, u)\right) \\
& \leqslant \frac{D_{x} k_{\varepsilon} \cdot p+D_{u} k_{\varepsilon}|p|^{2}}{\left(1+|p|^{2}\right)^{1 / 2}}+g n k_{\varepsilon}^{1+1 / n}+g n C \varepsilon
\end{aligned}
$$

for some positive constant $C$ depending on the Lipschitz constant of $k$ in $\bar{\Omega} \times[-R, R]$. Then, for $\varepsilon$ small, we have $\alpha-g n C \varepsilon>\frac{\alpha}{2}$ and we conclude.

We explicitly remark that if $k$ is Lipschitz continuous and satisfies (H1) then (H8) is clearly satisfied a.e. for some $\alpha>0$. Moreover in this case the existence and uniqueness of a continuous solution follows from Corollary 4.1. Thus in view of Corollary 5.1 in order to prove the Lipschitz regularity of the solution it is enough to verify that $\left(u_{\varepsilon}\right)$ is equibounded and the condition (5.14) holds. This is the purpose of the following

Theorem 5.2 (The strict monotone case). Assume (H1)-(H4), (H7), $\varphi \in C^{1,1}(\partial \Omega)$. Then there exists a unique Lipschitz continuous viscosity solution $u$ of (DP).

Proof. For all $\varepsilon>0$ small, let $u_{\varepsilon}$ be a solution of (DP) $)_{\varepsilon}$. The existence of a continuous solution $u_{\varepsilon}$ of $(\mathrm{DP})_{\varepsilon}$ follows from Corollary 4.1, because for $\varepsilon$ small $k_{\varepsilon}$ satisfies (H1)-(H4), (we observe that $\left.\left\|k_{\varepsilon}\right\|_{\infty} \leqslant\|k\|_{\infty}\right)$. The family $\left(u_{\varepsilon}\right)$ is equibounded in $\bar{\Omega}$ by a positive constant $M_{0}$ because for $\varepsilon$ small we have $\underline{v} \leqslant u_{\varepsilon} \leqslant \bar{v}$, where $\underline{v}, \bar{v}$ are the functions defined in Corollary 4.1. Because of Corollary 5.1 in order to prove that the family $\left(u_{\varepsilon}\right)$ is equicontinuous, it is enough to show that the condition (5.14) is satisfied for some $C>0$ independent of $\varepsilon$. Let us consider the function $\bar{u}$ and $\bar{u}$ defined in Lemma 5.1. In Lemma 5.1 it is shown that for $\lambda$ large $\bar{u}$ is a supersolution of (DP) and $\underline{u}$ is a strict super subsolution of (DP) in $\Omega_{\gamma}$ for some $\gamma>0$ small. Moreover they are Lipschitz continuous in $\Omega_{\gamma}$ with $\|D \bar{u}\|_{\infty},\|D \bar{u}\|_{\infty} \leqslant \bar{C}$, for some $\bar{C}>0$ depending on $\lambda$. One can readily see that if $\varepsilon>0$ is small then $\bar{u}$ and $\bar{u}$ are super and subsolutions of (DP) $)_{\varepsilon}$ as well. If we take $\lambda>\left(M_{0}+\|\varphi(x)\|_{\infty}\right) / \gamma$, then $\bar{u}(x) \leqslant u_{\varepsilon}(x) \leqslant \bar{u}(x)$ for all $x$ such that $d(x, \partial \Omega)=\gamma$. Thus by Theorem 4.1 we have $\bar{u}(x) \leqslant u_{\varepsilon}(x) \leqslant \bar{u}(x)$ in $\bar{\Omega}_{\gamma}$.

Take $(x, w) \in \partial(\Omega \times \Omega)$ and suppose that $x \in \partial \Omega$. There are two possibilities: either $d(w, \partial \Omega) \leqslant \gamma$ or $d(w, \partial \Omega)>\gamma$. If $d(w, \partial \Omega) \leqslant \gamma$ then

$$
u_{\varepsilon}(x)-u_{\varepsilon}(w) \leqslant \bar{u}(x)-\underline{u}(w) \leqslant \bar{C}|x-w| .
$$

If $d(w, \partial \Omega)>\gamma$ then $|x-w|>\gamma$ and therefore

$$
u_{\varepsilon}(x)-u_{\varepsilon}(w) \leqslant 2\left\|u_{\varepsilon}\right\|_{\infty} \leqslant 2\left\|u_{\varepsilon}\right\|_{\infty} \frac{|x-w|}{\gamma} .
$$

We observe that the Lipschitz constant of the solution $u_{\varepsilon}$ depends on the Lipschitz constant of the barriers $\bar{u}, \bar{u}$, on the function $k$ and on the $L^{\infty}$-norm of the solution $u_{\varepsilon}$. Thus if we choose $C=\max \left(\bar{C}, 2 M_{0} / \gamma\right)$, the condition 
(5.14) holds. Since (H8) hold a.e., the conclusion follows from Corollary 5.1 and by the uniqueness of a viscosity solution of (DP) proved in Corollary 4.1.

Proof of Theorem 1.1. The proof is contained in Corollary 4.1 and in Theorem 5.2.

In the general case, namely when $k$ is not strictly monotone with respect to the $u$ variable, we prove the existence by approximating the operator $F$ by a sequence of operators $F^{\varepsilon}$ which are strictly monotone with respect to $u$. More precisely for all $\varepsilon>0$ we define

$$
k^{\varepsilon}(x, u)=\varepsilon q(u)+k(x, u) ;
$$

where $q: \mathbb{R} \rightarrow[0,+\infty)$ is a bounded function of class $C^{1}$ such that $q^{\prime}>0$. We consider

$$
F^{\varepsilon}(x, u, p, X):= \begin{cases}\left(k^{\varepsilon}(x, u)\right)^{1 / n} f^{1 / n}(p)-(\operatorname{det} A(p, X))^{1 / n}, & \text { if } A(p, X) \geqslant 0, \\ +\infty, & \text { otherwise }\end{cases}
$$

and the Dirichlet problem

$$
\begin{cases}F^{\varepsilon}\left(x, u, D u, D^{2} u\right)=0 & \text { in } \Omega, \\ u(x)=\varphi(x) & \text { in } \partial \Omega,\end{cases}
$$

From Theorem 4.1 it follows that for all $\varepsilon>0$ there is a unique viscosity solution of (DP) ${ }^{\varepsilon}$ which is Lipschitz continuous in $\bar{\Omega}$ by Theorem 5.2. The main goal is to show that the family $\left(u^{\varepsilon}\right)_{\varepsilon}$ is equibounded and equicontinuous in $\bar{\Omega}$. We denote

$$
\widetilde{F}^{\varepsilon}(x, u, p, X)=-\operatorname{det}(A(p, X))+k^{\varepsilon}(x, p) f(p) .
$$

We start with the following lemma which is the analogous of Lemma 5.2.

Lemma 5.3. Assume $k \in C^{1}$ satisfies (H8). Then, for all $\varepsilon>0$ small, $k^{\varepsilon}$ satisfies the condition (1.8) for some positive $\alpha_{\varepsilon}$ depending on $\varepsilon$.

Proof. We set

$$
I^{\varepsilon}(x, u, p)=\frac{D_{x} k^{\varepsilon} \cdot p+D_{u} k^{\varepsilon}|p|^{2}}{\left(1+|p|^{2}\right)^{1 / 2}}+g n\left(k^{\varepsilon}\right)^{1+1 / n}=\frac{D_{x} k \cdot p+\left[D_{u} k+\varepsilon q^{\prime}(u)\right]|p|^{2}}{\left(1+|p|^{2}\right)^{1 / 2}}+g n\left(k^{\varepsilon}\right)^{1+1 / n} .
$$

Since $q, q^{\prime}>0$, if $L>1$ is large enough we have

$$
I_{\varepsilon}(x, u, p) \geqslant I(x, u, p)+\varepsilon \inf q^{\prime} / 2+g n(\varepsilon \inf q)^{1+1 / n} \geqslant \varepsilon \inf q^{\prime} / 2+g n(\varepsilon \inf q)^{1+1 / n} .
$$

Hence the conclusion follows by choosing for instance $\alpha_{\varepsilon}=\varepsilon \inf \left(q^{\prime}\right) / 4$ and by arguing exactly as in the proof of Lemma 5.2.

Next we show that the family $u^{\varepsilon}$ is equibounded and verifies (5.9) with some constant independent of $\varepsilon$.

Proposition 5.2. Assume (H3)-(H5), (H7), $\varphi \in C^{1,1}(\partial \Omega)$. Then there is a constant $K>0$ such that for all $\varepsilon$ small we have

(i) $\left\|u^{\varepsilon}\right\|_{\infty} \leqslant K$;

(ii) $\left|u^{\varepsilon}(x)-u^{\varepsilon}(y)\right| \leqslant K|x-y|$, for all $(x, y) \in \partial(\Omega \times \Omega)$.

Proof. To show (i) it is enough to observe that the functions $\underline{v}$ and $\bar{v}$ which have been considered in Corollary 4.1 are still sub and supersolutions of (DP) ${ }^{\varepsilon}$. Indeed we observe that by (H4) the function $\underline{v}$ is a strict subsolution of 
$F=0$, thus since $q$ is bounded, for all $\varepsilon$ small enough we have $F^{\varepsilon} \leqslant 0$. The function $\bar{v}$ is still a supersolution $F^{\varepsilon}=0$, since $q$ is positive.

The second property (ii) is a consequence of the fact that the functions $\bar{u}$ and $\bar{u}$ built in Lemma 5.1 are still local barriers for (DP $)^{\varepsilon}$. The proof of this claim follows again from the facts that the function $\bar{u}$ is a strict subsolution of $F=0$ and the function $q$ is positive and bounded. Thus the conclusion follows by the same arguments of the proof of Theorem 5.2.

By combining Corollary 5.1, Lemma 5.3 and Proposition 5.2 it follows

Corollary 5.2. Assume (H3)-(H5), (H7)-(H8), $\varphi \in C^{1,1}(\partial \Omega)$. For all $\varepsilon>0$ let $u^{\varepsilon}$ be the unique viscosity solution of $(\mathrm{DP})^{\varepsilon}$. Then there is a constant $C>0$ depending on $K, L,\|k\|_{\infty},(K, L$ being the constant appearing respectively in Proposition 5.2 and in condition $(\mathrm{H} 8)$ ) such that for $\varepsilon$ small we have

$$
\left|u^{\varepsilon}(x)-u^{\varepsilon}(y)\right| \leqslant C|x-y|, \quad \text { for all }(x, y) \in \Omega \times \Omega .
$$

From Proposition 5.2 and Corollary 5.2 it follows that the family $u^{\varepsilon}$ is equicontinuous and equibounded in $\bar{\Omega}$. Thus by applying Ascoli-Arzelà theorem we get the existence of a Lipschitz continuous solution of (DP) also in the case that $k$ satisfies (H5). More precisely we have

Theorem 5.3. Assume (H3)-(H5) and $(\mathrm{H} 7)-(\mathrm{H} 8) \varphi \in C^{1,1}(\partial \Omega)$. Then there exits a Lipschitz continuous solution of (DP).

Proof. For all $\varepsilon>0$ let $u^{\varepsilon}$ be the unique viscosity solution of (DP) ${ }^{\varepsilon}$. From Proposition 5.2 and Corollary 5.2 it follows that the family $u^{\varepsilon}$ is equicontinuous and equibounded in $\bar{\Omega}$. Thus by applying Ascoli-Arzelà theorem there is a subsequence $u_{\varepsilon_{j}}$ which converge uniformly as $j \rightarrow \infty$ to a function $u$ which is Lipschitz continuous in $\bar{\Omega}$. Since $F^{\varepsilon}$ converges locally uniformly to $F$, by the stability of viscosity solutions with respect to the uniformly convergence of $F^{\varepsilon}$ to $F$, we get that $u$ is a viscosity solution of (DP) and we conclude.

Proof of Theorem 1.3. The proof is contained in Theorems 5.3 and 4.3.

\section{Nonexistence results on balls}

In this section we present some nonexistence results which show that condition (H4) cannot be significantly relaxed when the domain $\Omega$ is a ball. We will denote by $v(x)$ the inner normal vector to $\partial \Omega$ at $x \in \partial \Omega$. First of all, by following the argument in [6, Theorem 1] and in [19, Corollary 1.1], we easily have

Proposition 6.1. Let $B=B(R) \subset \mathbb{R}^{N}$ be a ball of radius $R$ and let $u \in C^{0,1}(\bar{B})$ be a viscosity solution of $F=0$. Then necessarily

$$
R \leqslant \sup _{\Omega \times \mathbb{R}}(1 / k)^{1 / n}
$$

Proof. For $0<r<R$ we have that

$$
\phi(x)=C-\left(r^{2}-|x|^{2}\right)^{1 / 2}
$$


is in $C^{2}(B(r))$, and $\frac{\partial \phi}{\partial v}$ is $-\infty$ on the boundary. Since $u \in C^{0,1}(\bar{B})$ then $u-\phi$ has a maximum point at an interior point $x_{0} \in B(r)$. By definition of a viscosity solution of $F=0$, we have that $F\left(x_{0}, u\left(x_{0}\right), D \phi\left(x_{0}\right), D^{2} \phi\left(x_{0}\right)\right) \leqslant 0$, i.e.

$$
k\left(x_{0}, u\left(x_{0}\right)\right) \leqslant \frac{\operatorname{det} A\left(D \phi, D^{2} \phi\right)}{f(D \phi)}=\frac{1}{r^{n}},
$$

for all $r<R$. By letting $r \rightarrow R$ we get (6.1).

The estimate (6.1) can be obviously regarded as a first nonexistence result: If (6.1) does not hold, then we cannot find a viscosity solution $u \in C^{0,1}(\bar{\Omega})$ of $F=0$. We shall prove a stronger result when $\Omega$ is a ball. Our main tool is the following variant of the comparison principle.

Proposition 6.2. Let $\Omega \subset \mathbb{R}^{N}$ be a bounded domain and $\Gamma \subseteq \partial \Omega$ be relatively open and of class $C^{1}$. If $u \in$ $C(\bar{\Omega}) \cap C^{0,1}(\Omega \cup \Gamma)$ is a viscosity solution of $F \leqslant 0$ and $v \in C(\bar{\Omega}) \cap C^{0,1}(\Omega)$ is a viscosity solution of $F>0$ such that for all $x \in \Gamma$

$$
\liminf _{t \rightarrow 0^{+}} \frac{v(x+t v(x))-v(x)}{t}=-\infty \quad \text { on } \Gamma
$$

and $u \leqslant v$ in $\partial \Omega \backslash \Gamma$, then $u \leqslant v$ in $\Omega$.

Proof. By the comparison principle we have $\sup _{\Omega}(u-v) \leqslant \sup _{\Gamma}(u-v)^{+}$, but on $\Gamma$ we have

$$
\liminf _{t \rightarrow 0^{+}} \frac{v(x+t v(x))-v(x)}{t}=-\infty .
$$

Hence, $u-v$ cannot achieve a maximum value on $\Gamma$. Then $u \leqslant v$ on $\partial \Omega$.

Now assume that $k>0$ is independent of $u$ and $u \in C^{0,1}(\bar{B}(R))$ is a viscosity solution of $F=0$ in $B(R)$. Assume that there is a point $\xi_{0} \in \partial B$ such that

$$
k\left(\xi_{0}\right)>\frac{1}{R^{n}} .
$$

It is not restrictive to assume $\xi_{0}=0$ and that the interior unit normal to $\partial B$ at $\xi_{0}$ is $\left(0_{n}, 0_{n}, 1\right)$. We shall show that the boundary value of the function $u$ cannot be arbitrarily on $\xi_{0}$. By the continuity of $k$ we can assume that (6.2) holds in a neighborhood of $\xi_{0}$. In particular there is a positive $a<R$ such that (6.2) holds in $B_{a}=\{(x, y, t) \in B: t<a\}$. Next define the function

$$
w(x, y, t)=m+\psi(t),
$$

where $m=\sup _{\partial B \backslash B_{a}} u, \psi \in C^{2}((a, 2 R))$ is such that $\psi(2 R)=0, \psi^{\prime} \leqslant 0, \psi^{\prime}(a)=-\infty$. In $B \backslash \overline{B_{a}}$ we have that $F\left(\xi, w, D w, D^{2} w\right)=k(\xi)>0$. By Proposition 6.2 we have

$$
\sup _{B \backslash B_{a}} u \leqslant m+\psi(a) .
$$

Now we consider

$$
w_{a}(\xi)=m_{a}-\left(R^{2}-\left|\xi-\xi^{\prime}\right|^{2}\right)^{1 / 2}+M_{a}
$$

with $m_{a}=\sup _{B \cap\{t=a\}} u, M_{a}=\sup _{B \cap\{t=a\}}\left(R^{2}-\left|\xi-\xi^{\prime}\right|^{2}\right)^{1 / 2}$ and $\xi^{\prime}$ is the center of the ball $B$. In $B_{a}$ we have $F\left(\xi, w_{a}, D w_{a}, D^{2} w_{a}\right)=k(\xi)-R^{-n}>0$. Proposition 6.2 yields that

$$
\sup _{B_{a}} u \leqslant m_{a}+M_{a} .
$$


Finally, by applying estimate (6.3) to $m_{a}$ in (6.4) we obtain

$$
u\left(\xi_{0}\right) \leqslant m+\psi(a)+M_{a}=\sup _{\partial B \backslash B_{a}} u+\psi(a)+M_{a} .
$$

We remark that $\lim _{a \rightarrow 0} M_{a}=0$ and that we can choose $\psi$ such that $\lim _{a \rightarrow 0} \psi(a)=0$ [13, Eq. (14.67), p. 348]. Hence the estimate (6.5) shows that $u$ cannot be prescribed arbitrarily on $\partial \Omega$. Thus we have proved the following nonexistence theorem.

Theorem 6.1 (Nonexistence result on balls). Assume $k>0$ is independent of $u$ and there is a point $\xi_{0} \in \partial B$ such that (6.2) holds. Then there is $\varphi \in C^{\infty}(\bar{B})$ such that the Dirichlet problem (DP) (or (1.2)) is not solvable in the class of Lipschitz continuous viscosity solution.

\section{References}

[1] M. Bardi, I. Capuzzo Dolcetta, Optimal Control and Viscosity Solutions of Hamilton-Jacobi-Bellman Equations, Birkhäuser, Boston, 1997.

[2] G. Barles, Solutions de viscosite des equations de Hamilton-Jacobi, Collection "Mathematiques et Applications" de la SMAI, vol. 17, Springer-Verlag, 1994.

[3] G. Barles, A weak Bernstein method for fully nonlinear elliptic equations, Differential Integral Equations 4 (2) (1991) $241-262$.

[4] G. Barles, F. Da Lio, Remarks on the Dirichlet and state-constraint problems for quasilinear parabolic equations, Adv. Differential Equations 8 (2003) 897-922.

[5] G. Barles, E. Rouy, P.E. Souganidis, Remarks on the Dirichlet problem for quasilinear elliptic and parabolic equations, in: W.M. McEneaney, G.G. Yin, Q. Zhang (Eds.), Stochastic Analysis, Control, Optimization and Applications. A Volume in Honor of W.H. Fleming, Birkhäuser, Boston, 1999, pp. 209-222.

[6] E. Bedford, B. Gaveau, Hypersurfaces with bounded Levi form, Indiana Univ. J. 27 (5) (1978) 867-873.

[7] G. Citti , E. Lanconelli, A. Montanari, Smoothness of Lipschitz continuous graphs with non vanishing Levi curvature, Acta Math. 188 (2002) $87-128$.

[8] M.G. Crandall, P.L. Lions, Viscosity solutions of Hamilton-Jacobi-Bellman equations, Trans. Amer. Math. Soc. 277 (1983) 1-42.

[9] M.G. Crandall, H. Ishii, P.L. Lions, User's guide to viscosity solutions of second order partial differential equations, Bull. Amer. Soc. 27 (1992) 1-67.

[10] F. Da Lio, Strong comparison results for quasilinear equations in annular domains and applications, Comm. Partial Differential Equations 27 (1\&2) (2002) 283-323.

[11] J.P. D’ Angelo, Several Complex Variables and the Geometry of Real Hypersurfaces, Stud. Adv. Math., CRC Press, Boca Raton, FL, 1993.

[12] A. Debiard, B. Gaveau, Problème de Dirichlet pour l'équation de Lévi, Bull. Sci. Math. (2) 102 (4) (1978) 369-386.

[13] D. Gilgarg, N.S. Trudinger, Elliptic partial differential equations of second order, Grundlehrer Math. Wiss., vol. 224, Springer-Verlag, New York, 1977.

[14] L. Hörmander, An Introduction to Complex Analysis in Several Variables, Von Nostrand, Princeton, NJ, 1966.

[15] H. Ishii, Perron's method for Hamilton-Jacobi equations, Duke Math. J. 55 (1987) 369-384.

[16] H. Ishii, P.L. Lions, Viscosity solutions of fully nonlinear second-order elliptic partial differential equations, J. Differential Equations 83 (1) (1990) 26-78.

[17] L. Hörmander, Notions of Convexity, Progr. Math., vol. 127, Birkhäuser, Boston, 1994.

[18] S.G. Krantz, Function Theory of Several Complex Variables, second ed., AMS Chelsea Publishing, Providence, RI, 2001.

[19] A. Montanari, E. Lanconelli, Pseudoconvex fully nonlinear partial differential operators. Strong comparison theorems, J. Differential Equations 202 (2) (2004) 306-331.

[20] A. Montanari, F. Lascialfari, The Levi Monge-Ampère equation: smooth regularity of strictly Levi convex solutions, J. Geom. Anal. 14 (2) (2004) 331-353.

[21] R.M. Range, Holomorphic Functions and Integral Representation Formulas in Several Complex Variables, Springer-Verlag, New York, 1986.

[22] Z. Slodkowski, G. Tomassini, Weak solutions for the Levi equation and envelope of holomorphy, J. Funct. Anal. 101 (2) (1991) $392-407$.

[23] Z. Slodkowski, G. Tomassini, The Levi equation in higher dimensions and relationships to the envelope of holomorphy, Amer. J. Math. 116 (2) (1994) 479-499.

[24] N.S. Trudinger, The Dirichlet Problem for the prescribed curvature equations, Arch. Rational Mech. Anal. 111 (2) (1990) $153-179$. 